\title{
CONNECTIVITY, HOMOTOPY DEGREE, AND OTHER PROPERTIES OF $\alpha$-LOCALIZED WAVELETS ON $\mathbb{R}$
}

\author{
Gustavo Garrigós
}

\begin{abstract}
In this paper, we study general properties of $\alpha$-localized wavelets and multiresolution analyses, when $\frac{1}{2}<\alpha \leq \infty$. Related to the latter, we improve a well-known result of A. Cohen by showing that the correspondence $m \mapsto \widehat{\varphi}=\prod_{1}^{\infty} m\left(2^{-j} \cdot\right)$, between lowpass filters in $H^{\alpha}(\mathbb{T})$ and Fourier transforms of $\alpha$-localized scaling functions (in $H^{\alpha}(\mathbb{R})$ ), is actually a homeomorphism of topological spaces. We also show that the space of such filters can be regarded as a connected infinite dimensional manifold, extending a theorem of A. Bonami, S. Durand and G. Weiss, in which only the case $\alpha=\infty$ is treated. These two properties, together with a careful study of the "phases" that give rise to a wavelet from the MRA, will allow us to prove that the space $\mathcal{W}_{\alpha}$, of $\alpha$-localized wavelets, is arcwise connected with the topology of $L^{2}\left(\left(1+|x|^{2}\right)^{\alpha} d x\right)$ (modulo homotopy classes). This last result is new even for the case $\alpha=\infty$, as well as the considerations about the "homotopy degree" of a wavelet.
\end{abstract}

\section{Introduction}

A wavelet is a function $\psi \in L^{2}(\mathbb{R})$ such that the system

$$
\psi_{j, k}(x)=2^{j / 2} \psi\left(2^{j} x-k\right), \quad j, k \in \mathbb{Z},
$$

is an orthonormal basis for $L^{2}(\mathbb{R})$. Elementary properties, examples, and standard notation can be found in the texts: [5] and [7] (Part II).

Keywords. Wavelet, MRA, Sobolev space, homotopy degree, phase.

1991 Mathematics subject classifications: 42C15. 
In this paper, we shall be interested in those wavelets having a certain smoothness and decay at infinity. The smoothness will be given in terms of Sobolev spaces, and the decay in terms of " $L^{2}$-localization".

We say that a measurable function $f: \mathbb{R} \rightarrow \mathbb{C}$ is $\alpha$-localized whenever

$$
\int_{\mathbb{R}}|f(x)|^{2}\left(1+|x|^{2}\right)^{\alpha} d x<\infty .
$$

A function $f$ for which the integral in (1.1) is finite for every $\alpha \in \mathbb{Z}^{+}$ is said to be $\infty$-localized or to have polynomial decay. We will suppose $\frac{1}{2}<\alpha \leq \infty$, so that, in particular, the Fourier transform of $f$, given by

$$
\hat{f}(\xi)=\int_{\mathbb{R}} f(x) e^{-i x \cdot \xi} d x, \quad \xi \in \mathbb{R},
$$

represents a continuous function in $\mathbb{R}$. Note, further, that a function $f$ is $\alpha$-localized if and only if $\hat{f}$ belongs to the Sobolev space $H^{\alpha}(\mathbb{R})$, since the expression in (1.1) is precisely $\|\hat{f}\|_{H^{\alpha}(\mathbb{R})}^{2}$.

In the sequel, we will consider the following class of wavelets:

Definition 1.2. Let $\frac{1}{2}<\alpha \leq \infty$. We say that $\psi \in \mathcal{W}_{\alpha}$ if

(i) $\psi$ is a wavelet.

(ii) $\psi$ is $\alpha$-localized (equivalently, $\widehat{\psi} \in H^{\alpha}(\mathbb{R})$ ).

(iii) There exists a positive $\varepsilon$ such that $\psi \in H^{\varepsilon}(\mathbb{R})$.

We endow $\mathcal{W}_{\alpha}$ with the topology of $L^{2}\left(\left(1+|x|^{2}\right)^{\alpha} d x\right)$ (respectively, of the Fréchet space $\cap_{k=1}^{\infty} L^{2}\left(\left(1+|x|^{2}\right)^{k} d x\right)$, if $\left.\alpha=\infty\right)$. That is, for a sequence $\psi, \psi_{1}, \psi_{2}, \ldots \in \mathcal{W}_{\alpha}$, the statement " $\psi_{n} \rightarrow \psi$ in $\mathcal{W}_{\alpha}$ ", means that " $\widehat{\psi}_{n} \rightarrow \widehat{\psi}$ in $H^{\alpha}(\mathbb{R})$ ".

In this setting, one of the main theorems in this paper can then be stated as follows:

Theorem 1.3. Let $\frac{1}{2}<\alpha \leq \infty$, and $\psi \in \mathcal{W}_{\alpha}$. Then there exists an integer $k=k(\psi)$ such that

$$
\int_{\mathbb{R}} x|\psi(x)|^{2} d x=k+\frac{1}{2} .
$$

Moreover, for each $k \in \mathbb{Z}$, the set

$$
\mathcal{W}_{\alpha}^{(k)}=\left\{\psi \in \mathcal{W}_{\alpha}: \int_{\mathbb{R}} x|\psi(x)|^{2} d x=k+\frac{1}{2}\right\}
$$

is a connected, open and closed topological subspace of $\mathcal{W}_{\alpha}$. In particular, $\mathcal{W}_{\alpha}$ can be written in connected components as the disjoint union: $\mathcal{W}_{\alpha}=$ $\cup_{k \in \mathbb{Z}} \mathcal{W}_{\alpha}^{(k)}$. 
As interesting as the result itself, is the technique we use to prove it, which includes a detailed study of the $\alpha$-localized multiresolution analyses (precise definitions will be postponed to subsequent sections). Recall that, by a theorem of P. G. Lemarié $([8])$, every wavelet in $\mathcal{W}_{\alpha}$ must arise from one of these MRA's. That is, given $\psi \in \mathcal{W}_{\alpha}$, there is an $\alpha$-localized scaling function $\varphi$, with associated low-pass filter $m$, and a unimodular $2 \pi$-periodic function $\nu$ (sometimes referred to as "phase") such that

$$
\widehat{\psi}(\xi)=e^{-i \xi / 2} \overline{\nu(\xi)} \overline{m(\xi / 2+\pi)} \widehat{\varphi}(\xi / 2), \quad \xi \in \mathbb{R} .
$$

The "mild" condition given in (iii) of Definition 1.2 plays a crucial role in the proof of Lemarié's theorem. However, it is not too restrictive since the converse is also true $([\mathbf{4}])$ : every $\alpha$-localized wavelet arising from an MRA satisfies (iii) and, therefore, belongs to $\mathcal{W}_{\alpha}$. This characterization for wavelets in $\mathcal{W}_{\alpha}$ produces a whole variety of examples, including those of Daubechies, Lemarié-Meyer, or the wavelets obtained in [6] by "smoothing" discontinuous low-pass filters. Moreover, general questions, like the connectedness of the set $\mathcal{W}_{\alpha}$, can be understood from their study on the different elements of an $\alpha$-localized MRA: filters, scaling functions, and phases.

In section 2 , we study the set, $\mathcal{E}_{\alpha}$, of low-pass filters associated with $\alpha$-localized scaling functions. We show that it is an arcwise connected manifold when endowed with the topology of the periodic Sobolev space $H^{\alpha}(\mathbb{T})$. This set was introduced and studied as a manifold for the first time by A. Bonami, S. Durand and G. Weiss $([\mathbf{1}])$, in the case $\alpha=\infty$. Our contribution here consists mainly in adapting the definitions and proofs for other values of $\alpha$.

In section 3 , we take care of the $\alpha$-localized scaling functions, showing that the set of all these is homeomorphically equivalent to $\mathcal{E}_{\alpha}$. Like in the $\alpha=\infty$ case, treated by the authors of [1], the homeomorphism is given by the usual assignment to each scaling function of its associated low-pass filter, which according to Cohen's theorem, can be written as:

$$
m \in \mathcal{E}_{\alpha} \longmapsto \widehat{\varphi}(\xi)=\prod_{j=1}^{\infty} m\left(2^{-j} \xi\right) .
$$

However, the machinery required to prove the continuity of this map when $\alpha<\infty$, is much more sophisticated than for the case $\alpha=\infty$. This is due, on the one hand, to the use of Sobolev norms, but also to the sharpness of the result, which is no longer true, for instance, if one enlarges slightly the domain (see Example 3.12 below). Convergence questions involving filters and scaling functions have also been considered by P. G. Lemarié in [9], although only in the $\alpha=\infty$ case, leaving open the study for other values of $\alpha$. 
In section 4, we turn to the study of the "phase" $\nu$ in (1.5), left aside by many authors in the wavelet literature, but key to obtain our general result in Theorem 1.3. In [1], for instance, only wavelets that admit the phase $\nu \equiv 1$ are considered and, therefore, only those can be shown to be connected by arcs. So, the general connectivity statement we present in Theorem 1.3 is new even in the case of the space $\mathcal{W}_{\infty}$. Formula (1.4), relating the homotopy degree of the phase, and the "center of mass" of the wavelet plays a central role here, since from it we can easily see that $\mathcal{W}_{\alpha}$ is not a connected topological space (with the topology considered in Definition 1.2). Indeed, any continuous path $t \mapsto \psi_{t}$ in $\mathcal{W}_{\alpha}$ must leave the integral in (1.4) constant and, therefore, a wavelet $\psi$ can never be joined to its shift $\psi(\cdot-1)$, continuously in $L^{2}(|x| d x)$. This formula was announced for the case $\alpha=\infty$ in $[\mathbf{8}]$, and amounts to the work of L. Villemoes ([11]) on compactly supported wavelets, but the simple and general proof we give here (which covers all the values of $\alpha>\frac{1}{2}$ ) seems to be new. To conclude section 4 , we classify wavelets according to the homotopy degree of their phases, and establish, then, our final connectivity result.

In the appendix, we study a bit further the decomposition of a wavelet in terms of a phase and a filter as in (1.5). This question was previously considered by P. G. Lemarié in [8], where it was proved that every wavelet $\psi \in \mathcal{W}_{\infty}^{(0)}$ can be written as:

$$
\widehat{\psi}(\xi)=c e^{-i \xi / 2} \overline{m(\xi / 2+\pi)} \prod_{j=2}^{\infty} m\left(2^{-j} \xi\right), \quad \xi \in \mathbb{R},
$$

with a filter $m \in C^{\infty}(\mathbb{T})$ and a unimodular constant $c \in \mathbb{C}$. In other words, a constant phase $\nu \equiv c$ is "admissible" in (1.5). These results are no longer true in the range $\frac{1}{2}<\alpha<\infty$, as we illustrate with different counter-examples. However, when $\psi \in \mathcal{W}_{\alpha}^{(0)}$, we show how to find a filter of maximum regularity: $m \in \cap_{\beta<\alpha} H^{\beta}(\mathbb{T})$, and how the choice of this filter is unique (up to multiplication by $e^{i \ell \xi}, \ell \in \mathbb{Z}$ ) only when $\alpha>1$.

Preliminary warning and acknowledgements. The content of this article forms part of the $\mathrm{Ph}$. D. thesis of the author $([\mathbf{3}])$, completed in Washington University, St. Louis, in May 1998, under the direction of Guido Weiss. The thesis is available at http://www.math.wustl.edu/ gustavo, and it contains several technical results that are (only) referred to in this presentation. We have not included this material here since it also appears in $[\mathbf{1}]$ or $[\mathbf{7}]$; however, the reader might find the write-up in $[\mathbf{3}]$ more accessible since the notation and terminology are closer to the ones used in this article. 
The author is indebted to Guido Weiss for his constant support and advice during the completion of the thesis and the write-up of this article. We also wish to thank A. Bonami and M. Taibleson for some very useful remarks during different stages of this work.

\section{The space $\mathcal{E}_{\alpha}$ of low-pass filters}

We defined above $\mathcal{E}_{\alpha}$ as the set of low-pass filters associated with $\alpha$-localized scaling functions. Since in this section we won't use any of the terminology of multiresolution analyses, but instead will concentrate only on topological and manifold-like properties of $\mathcal{E}_{\alpha}$, we might as well consider the equivalent definition of this set given in terms of Cohen's Theorem. That is, a function $m$ is in $\mathcal{E}_{\alpha}$ if and only if belongs to the Sobolev space $H^{\alpha}(\mathbb{T})$ and satisfies the conditions:

(2.1) $m(0)=1$.

(2.2) $|m(\xi)|^{2}+|m(\xi+\pi)|^{2}=1, \quad \xi \in \mathbb{R}$.

(2.3) $\exists K \subset \mathbb{R}$ compact such that $0 \in \stackrel{\circ}{K}, \sum_{\ell \in \mathbb{Z}} \chi_{K}(\xi+2 \ell \pi)=1$, a.e. $\xi \in \mathbb{R}$, and $m\left(2^{-j} \xi\right) \neq 0, j \geq 1, \xi \in K$.

Of course, as a topological space $\mathcal{E}_{\alpha}$ will be endowed with the topology of $H^{\alpha}(\mathbb{T})$. Our goal is to establish the following result:

Theorem 2.4. Let $\frac{1}{2}<\alpha \leq \infty$. Then $\mathcal{E}_{\alpha}$ is an infinite dimensional manifold, in the sense that for every $m \in \mathcal{E}_{\alpha}$ there exists a neighborhood of $m, U \subset \mathcal{E}_{\alpha}$, a neighborhood of $\mathbf{0}, V \subset H^{\alpha}(\mathbb{T})$, and a homeomorphism

$$
\Phi: U \rightarrow V, \quad \text { such that } \Phi(m)=\mathbf{0}
$$

Moreover, $\mathcal{E}_{\alpha}$ is an arcwise connected topological space.

The proof of this theorem is not much different to the one presented by A. Bonami et al. in [1], for the case $\alpha=\infty$. For the sake of brevity, and in order to move quickly to the new material in subsequent sections, we just indicate how to adapt the arguments in $[\mathbf{1}]$ to the case $\frac{1}{2}<\alpha<\infty$. The reader interested in more details is referred to the monograph $[\mathbf{3}]$.

Before getting started, however, we would like to illustrate with an example (also from [1]) the intuitive idea underlying Theorem 2.4.

Example 2.5. Trigonometric polynomials of degree $\leq 3$ in $\mathcal{E}_{\alpha}$. 
Consider the family of functions

$$
m_{(a, c)}(\xi)=\frac{1+e^{i \xi}}{2}\left(a+b e^{i \xi}+c e^{i 2 \xi}\right), \quad \xi \in \mathbb{R},
$$

where $a, b, c$ are real numbers such that

$$
b=1-a-c \quad \text { and } a^{2}+c^{2}=a+c .
$$

An easy computation shows that (2.1) and (2.2) hold for $m_{(a, c)}$, if and only if the triad $(a, b, c)$ is taken as in (2.7). These equalities can also be interpreted as $(a, c)$ lying in a circle $C=\left\{(a, c) \in \mathbb{R}^{2}:\left(a-\frac{1}{2}\right)^{2}+\left(c-\frac{1}{2}\right)^{2}=\right.$ $\left.\frac{1}{2}\right\}$ (see Figure 2.1, below).

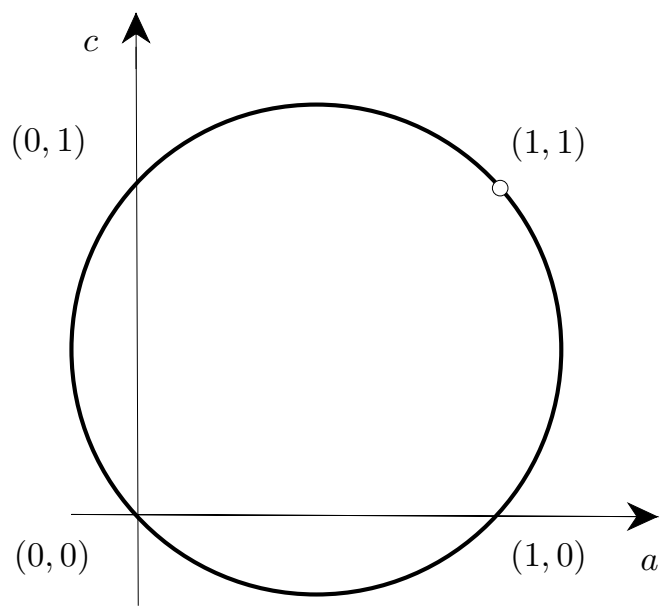

Figure 2.1. Circle $C$ of low-pass filters in Example 2.5.

When $(a, c)=(1,1)$ we obtain

$$
m_{(1,1)}(\xi)=\frac{1+e^{i 3 \xi}}{2},
$$

which is known not to belong to $\mathcal{E}_{\alpha}$ (see Example $\mathrm{F}$ in Chapter 2 of [5]). On the other hand, when $(a, c) \in C \backslash\{(1,1)\}$, an elementary calculation shows that, with the choice $K=[-\pi, \pi], m_{(a, c)}$ satisfies Cohen's condition (2.3) and, hence, belongs to $\mathcal{E}_{\alpha}$. Clearly, the set $\left\{m_{(a, c)},(a, c) \in C \backslash\{(1,1)\}\right\}$ is a connected topological space with a 1-dimensional manifold structure.

We now concentrate in the proof of Theorem 2.4, which will be splitted into two parts: the manifold condition, and the connectivity property. 


\subsection{The manifold condition.}

Following the ideas in [1] , we denote by $\mathcal{F}_{\alpha}$ the topological subspace of $H^{\alpha}(\mathbb{T})$ whose elements satisfy $(2.1)$ and $(2.2)$. That is, we have removed from the space $\mathcal{E}_{\alpha}$ the intricate condition (2.3). Then we have the following proposition.

Proposition 2.8. Let $\alpha>\frac{1}{2}$. Then $\mathcal{E}_{\alpha}$ is an open subset of $\mathcal{F}_{\alpha}$.

Once Proposition 2.8 is proved, it will suffice to show that $\mathcal{F}_{\alpha}$ is a manifold to establish the first part of Theorem 2.4. But this can be done proceeding exactly as in the proof of Theorem 2.1 of [1] , after replacing the space $C^{\infty}(\mathbb{T})$ by $H^{\alpha}(\mathbb{T})$, and noticing that the last one is a Banach algebra (when $\alpha>\frac{1}{2}$ ) and hence, closed under multiplication and the action of holomorphic functions (that is, $f \in H^{\alpha}(\mathbb{T})$ and $F$ holomorphic in a neighborhood of $f(\mathbb{T})$, imply that $F \circ f \in H^{\alpha}(\mathbb{T})$ ). The verification of these facts is left to the interested reader (who may consult [3] for further details). Note that as an immediate corollary of our results we obtain:

Corollary 2.9. Let $\alpha>\frac{1}{2}$. Then $\mathcal{E}_{\alpha}$ and $\mathcal{F}_{\alpha}$ are locally connected topological spaces.

The proof of Proposition 2.8, however, requires some more modifications, and we present it here for completeness. In fact, it is an immediate consequence of the following lemma, and the Sobolev Embedding Theorem. We will denote by $\Lambda^{\beta}(\mathbb{T})$ the usual Lipschitz space on the torus, $0<\beta<1$.

Lemma 2.10. Let $\alpha>\frac{1}{2}$ and $0<\beta<\min \left\{1, \alpha-\frac{1}{2}\right\}$. Let $m \in \mathcal{F}_{\alpha}$, $K$ be a compact set on $\mathbb{R}$, and $\varepsilon>0$. Then there exists a positive $\delta=\delta(\beta, \varepsilon, K)$ such that for every $F \in \mathcal{F}_{\alpha}$ with $\|F-m\|_{\Lambda^{\beta}(\mathbb{T})}<\delta$ we have

$$
\left|\prod_{j=1}^{\infty} F\left(2^{-j} \xi\right)-\prod_{j=1}^{\infty} m\left(2^{-j} \xi\right)\right|<\varepsilon, \quad \text { for all } \xi \in K .
$$

Proof: Let us denote by

$$
\widehat{\varphi}_{F}(\xi)=\prod_{j=1}^{\infty} F\left(2^{-j} \xi\right) \quad \text { and } \quad \widehat{\varphi}(\xi)=\prod_{j=1}^{\infty} m\left(2^{-j} \xi\right), \quad \xi \in \mathbb{R} .
$$


It is well-known that the infinite products above converge uniformly on compact sets and represent continuous functions in $\mathbb{R}$ (see, e.g., Lemma 4.5 in Chapter 7 of $[\mathbf{5}]$ ). Now, note that for every $\xi \in \mathbb{R}$,

$(2.11) \widehat{\varphi}_{F}(\xi)-\widehat{\varphi}(\xi)=\sum_{\ell=1}^{\infty}\left[\prod_{j=1}^{\ell-1} m\left(2^{-j} \xi\right)\right]\left[F\left(2^{-\ell} \xi\right)-m\left(2^{-\ell} \xi\right)\right] \widehat{\varphi}_{F}\left(2^{-\ell} \xi\right)$,

where the series converges uniformly and absolutely on $K$. Indeed, the partial sums of the series satisfy

$$
\begin{aligned}
& \sum_{\ell=1}^{N}\left[\prod_{j=1}^{\ell-1} m\left(2^{-j} \xi\right)\right]\left[F\left(2^{-\ell} \xi\right)-m\left(2^{-\ell} \xi\right)\right] \widehat{\varphi}_{F}\left(2^{-\ell} \xi\right) \\
= & \widehat{\varphi}_{F}(\xi)-\left[\prod_{j=1}^{N} m\left(2^{-j} \xi\right)\right] \widehat{\varphi}_{F}\left(2^{-N} \xi\right) \rightarrow \widehat{\varphi}_{F}(\xi)-\widehat{\varphi}(\xi), \quad \text { as } N \rightarrow \infty,
\end{aligned}
$$

the convergence in the last step following from properties of the infinite product and the continuity of $\widehat{\varphi}_{F}$ at $\xi=0$. Suppose now that the compact set $K \subset[-M, M]$, for some $M>0$, and let $j_{0}$ be a positive integer such that $2^{-j_{0}} M \leq \pi$. Then, for all $\xi \in K$, and using that $|F|,|m| \leq 1, F(0)=m(0)=1$, we have:

$$
\begin{aligned}
\left|\widehat{\varphi}_{F}(\xi)-\widehat{\varphi}(\xi)\right| & \leq \sum_{\ell=1}^{\infty}\left|F\left(2^{-\ell} \xi\right)-m\left(2^{-\ell} \xi\right)\right| \\
& \leq \sum_{\ell=1}^{j_{0}}\|F-m\|_{\infty}+\sum_{\ell=j_{0}+1}^{\infty}\left|2^{-\ell} \xi\right|^{\beta}\|F-m\|_{\Lambda^{\beta}(\mathbb{T})} .
\end{aligned}
$$

Then, if we take $\delta<\frac{\varepsilon}{2 j_{0}}$ and $\delta<\frac{\varepsilon\left(2^{\beta}-1\right)}{2(2 M)^{\beta}}$, we have $\left|\widehat{\varphi}_{F}(\xi)-\widehat{\varphi}(\xi)\right|<\varepsilon$.

\subsection{The connectivity.}

To end this section, we need to show that both $\mathcal{F}_{\alpha}$ and $\mathcal{E}_{\alpha}$ are connected, establishing then Theorem 2.4. But the proof of this fact, once again, does not differ much from the $\alpha=\infty$ case studied in [1]. There, it was shown that every filter (in $\mathcal{F}_{\infty}$, or $\mathcal{E}_{\infty}$ ) of the form

$$
m_{0}(\xi)=\frac{P_{0}(\xi)}{\sqrt{\left|P_{0}(\xi)\right|^{2}+\left|P_{0}(\xi+\pi)\right|^{2}}}, \quad \xi \in \mathbb{T}
$$


where $P_{0}$ is a trigonometric polynomial, can be joined with a continuous path:

$$
t \in[0,1] \longmapsto m_{t}=\frac{P_{t}}{\sqrt{\left|P_{t}\right|^{2}+\left|P_{t}(\cdot+\pi)\right|^{2}}},
$$

consisting of functions of the same type, to the Haar filter $m_{1}(\xi)=$ $\frac{1+e^{i \xi}}{2} \in \mathcal{E}_{\infty}$. This is essentially due to an argument using the Féjer-Riesz lemma for positive trigonometric polynomials (see section 3 of [1]).

From here and Corollary 2.9, the connectivity question reduces to show that the set of filters of the form (2.12) is dense in the spaces $\mathcal{F}_{\alpha}$ and $\mathcal{E}_{\alpha}$. The proof of this fact is simple, but again somewhat different from the one given in $[\mathbf{1}]$, so we present it below for completeness.

Proposition 2.13. Let $\alpha>\frac{1}{2}$ and $F \in \mathcal{F}_{\alpha}$. Then there exists a sequence of trigonometric polynomials $\left\{P_{n}\right\}_{n=1}^{\infty}$ such that $P_{n}(0)=1$, $P_{n}(\pi)=0, n=1,2, \ldots$ and

$$
\frac{P_{n}}{\sqrt{\left|P_{n}\right|^{2}+\left|P_{n}(\cdot+\pi)\right|^{2}}} \rightarrow F \quad \text { in } \mathcal{F}_{\alpha}, \quad \text { as } n \rightarrow \infty .
$$

Proof: Let $\left\{Q_{n}\right\}_{n=1}^{\infty}$ be a sequence of trigonometric polynomials such that $Q_{n} \rightarrow F$ in $H^{\alpha}(\mathbb{T})$ (e.g., take the $n^{\text {th }}$-symmetric partial sum of the Fourier series of $F$ ). Then, by the Sobolev Embedding Theorem:

$$
\lim _{n \rightarrow \infty} Q_{n}(0)=F(0)=1 \text { and } \lim _{n \rightarrow \infty} Q_{n}(\pi)=F(\pi)=0 .
$$

Therefore, for large $n$, we have $\left|Q_{n}(0)-Q_{n}(\pi)\right|>\frac{1}{2}$. Define, in these cases,

$$
P_{n}(\xi)=\frac{Q_{n}(\xi)-Q_{n}(\pi)}{Q_{n}(0)-Q_{n}(\pi)}, \quad \xi \in \mathbb{R} .
$$

Then, $P_{n}$ are trigonometric polynomials converging to $F$ in the topology of $H^{\alpha}(\mathbb{T})$. Moreover, $P_{n}(0)=1$ and $P_{n}(\pi)=0$. Once again, the Sobolev Embedding Theorem tells us that $\left|P_{n}(\xi)\right|^{2}+\left|P_{n}(\xi+\pi)\right|^{2} \rightarrow 1$, uniformly in $\mathbb{T}$, and therefore, for $n$ large enough we must have

$$
\left|P_{n}(\xi)\right|^{2}+\left|P_{n}(\xi+\pi)\right|^{2}>\frac{1}{2}, \quad \forall \xi \in \mathbb{T} .
$$

Now, the properties of the Banach algebra $H^{\alpha}(\mathbb{T})$ imply that

$$
\left(\left|P_{n}(\cdot)\right|^{2}+\left|P_{n}(\cdot+\pi)\right|^{2}\right)^{-1 / 2} \in H^{\alpha}(\mathbb{T})
$$

and, consequently, (2.14) holds.

Remark 2.15. Note that if $F \in \mathcal{E}_{\alpha}$, the trigonometric polynomials $\left\{P_{n}\right\}$ in the previous proposition can be taken to satisfy Cohen's condition (2.3). This follows from the fact that $\mathcal{E}_{\alpha}$ is an open subset of $\mathcal{F}_{\alpha}$, and implies in particular that the former set is connected. 


\section{The space $\mathcal{S}_{\alpha}$ of $\alpha$-localized scaling functions}

In this section we give a topological characterization of the $\alpha$-localized scaling functions in terms of the associated low-pass filters in $\mathcal{E}_{\alpha}$. We begin by recalling some precise definitions from the theory of multiresolution analyses (see Chapter 2 of [5], or Chapter 4 of [7]).

An $\alpha$-localized MultiResolution Analysis (MRA) is a sequence, $\left\{V_{j}\right\}_{j \in \mathbb{Z}}$, of closed subspaces of $L^{2}(\mathbb{R})$, such that

(i) $V_{j} \subset V_{j+1}, \quad \overline{\cup_{j} V_{j}}=L^{2}(\mathbb{R})$.

(ii) $f(x) \in V_{j} \Longleftrightarrow f(2 x) \in V_{j+1}$.

(iii) $\exists$ an $\alpha$-localized function $\varphi:\{\varphi(\cdot-k)\}_{k \in \mathbb{Z}}$ is an orthonormal basis for $V_{0}$.

A function $\varphi$ as in (iii) above is called an ( $\alpha$-localized) scaling function for the MRA. Without loss of generality we will assume that scaling functions associated with $\alpha$-localized MRA's satisfy $\widehat{\varphi}(0)=1$. We will denote by $\mathcal{S}_{\alpha}$ the set of all the Fourier transforms of $\alpha$-localized scaling functions, endowed with the topology of $H^{\alpha}(\mathbb{R})$. For simplicity, and unless stated otherwise, we will suppose $\frac{1}{2}<\alpha<\infty$. The results for $\alpha=\infty$ have already been treated in $[\mathbf{1}]$, and [9] (although they can also be obtained by iteration of our arguments).

Associated with each scaling function $\varphi$, there is a (unique) sequence $\left\{c_{k}\right\}_{k \in \mathbb{Z}}$ in $\ell^{2}(\mathbb{Z})$ such that

$$
\frac{1}{2} \varphi\left(\frac{x}{2}\right)=\sum_{k \in \mathbb{Z}} c_{k} \varphi(x-k), \quad \text { in } L^{2}(\mathbb{R}) .
$$

By taking Fourier transforms of both sides in (3.1), we obtain the scaling equation

$$
\widehat{\varphi}(2 \xi)=m(\xi) \widehat{\varphi}(\xi), \quad \text { a.e. } \xi \in \mathbb{R}
$$

where $m(\xi)=\sum_{k \in \mathbb{Z}} c_{k} e^{-i k \xi}$, is a $2 \pi$-periodic function in $L^{2}(\mathbb{T})$ called the low-pass filter associated with the scaling function $\varphi$, and the coefficients $c_{k}$ are given by the formula

$$
c_{k}=\int_{\mathbb{R}} \frac{1}{2} \varphi\left(\frac{x}{2}\right) \overline{\varphi(x-k)} d x, \quad k \in \mathbb{Z} .
$$

When $\varphi$ is $\alpha$-localized, it can be shown that $\sum_{k \in \mathbb{Z}}\left|c_{k}\right|^{2}\left(1+|k|^{2}\right)^{\alpha}<\infty$, and hence, $m \in H^{\alpha}(\mathbb{T})$. This is also a consequence of the following lemma, whose proof can be found in Chapter 3 of $[\mathbf{7}]$. 
Lemma 3.4. Let $f, g$ be $\alpha$-localized functions $\left(\alpha>\frac{1}{2}\right)$ and

$$
c_{k}=\int_{\mathbb{R}} f(x) g(x-k) d x, \quad k \in \mathbb{Z} .
$$

Then, there exists a constant $C=C(\alpha)>0$ such that

$$
\left[\sum_{k \in \mathbb{Z}}\left|c_{k}\right|^{2}\left(1+|k|^{2}\right)^{\alpha}\right]^{\frac{1}{2}} \leq C\|\hat{f}\|_{H^{\alpha}(\mathbb{R})}\|\hat{g}\|_{H^{\alpha}(\mathbb{R})} .
$$

\subsection{An extension of Cohen's Theorem.}

The original statement of Cohen's Theorem (see [2]) characterizes the low-pass filters corresponding to polynomial decay scaling functions, as the functions in $C^{\infty}(\mathbb{T})$ satisfying conditions (2.1), (2.2) and (2.3). In this case, the scaling function can be recovered from $m$ by the infinite product formula:

$$
\widehat{\varphi}(\xi)=\prod_{j=1}^{\infty} m\left(2^{-j} \xi\right)
$$

obtained, by iteration, from the scaling equation (3.2). This theorem was extended to the full range of $\alpha$-localized scaling functions, $\alpha>\frac{1}{2}$, by P. G. Lemarié (see Theorem 1 in Chapter 4 of [7]), and can be stated as follows:

Theorem 3.7 (Cohen, Lemarié). Let $\frac{1}{2}<\alpha \leq \infty$. Then,

(i) If $\widehat{\varphi} \in \mathcal{S}_{\alpha}$ then its associated low-pass filter belongs to $\mathcal{E}_{\alpha}$.

(ii) If $m \in \mathcal{E}_{\alpha}$ then the function given by the infinite product in (3.6) belongs to $\mathcal{S}_{\alpha}$.

The main result in this section is to show that this characterization is also topological:

Theorem 3.8. Let $\frac{1}{2}<\alpha \leq \infty$. Then the mapping

$$
\begin{aligned}
M: \mathcal{E}_{\alpha} & \longrightarrow \mathcal{S}_{\alpha} \\
m & \longmapsto \hat{\varphi}=\prod_{j=1}^{\infty} m\left(2^{-j} \cdot\right)
\end{aligned}
$$

is a homeomorphism of topological spaces. 
It follows directly from Cohen's Theorem that the map $M$ in Theorem 3.8 is a bijection. Moreover, it is not hard to show that the inverse map $M^{-1}$, defined via the Fourier coefficients in (3.3), is continuous, even when the domain consists of the whole space $H^{\alpha}(\mathbb{R})$ (in fact, from Lemma 3.4 we see that the "extension" of $M^{-1}$ as a bilinear operator from $H^{\alpha}(\mathbb{R}) \times H^{\alpha}(\mathbb{R})$ into $H^{\alpha}(\mathbb{T})$ is bounded). However, matters tend to become complicated when dealing with the direct map $M$. To illustrate better what we mean, let us note first the following property of the space $\mathcal{S}_{\alpha}$.

Lemma 3.9. Let $\frac{1}{2}<\alpha \leq \infty$. Then $\mathcal{S}_{\alpha}$ is closed in $H^{\alpha}(\mathbb{R})$.

Proof: Let $\left\{\widehat{\varphi}_{n}\right\}_{n=1}^{\infty}$ be a sequence in $\mathcal{S}_{\alpha}$ such that $\widehat{\varphi}_{n} \rightarrow \widehat{\varphi}$ in $H^{\alpha}(\mathbb{R})$. We need to see that $\varphi$ is a scaling function with $\widehat{\varphi}(0)=1$. The latter is a consequence of the Sobolev Embedding Theorem, since $\widehat{\varphi}(0)=$ $\lim _{n \rightarrow \infty} \widehat{\varphi}_{n}(0)=1$. To show the former, it is enough to check the following two conditions (see Chapter 7 in $[\mathbf{5}]$ ):

(i) $\sum_{k \in \mathbb{Z}}|\widehat{\varphi}(\xi+2 k \pi)|^{2}=1, \quad$ a.e. $\xi \in \mathbb{T}$.

(ii) There exists a $2 \pi$-periodic function $m \in L^{2}(\mathbb{T})$ such that

$$
\widehat{\varphi}(2 \xi)=m(\xi) \widehat{\varphi}(\xi), \quad \text { a.e. } \xi \in \mathbb{R} .
$$

Let us establish (i) first:

$$
\begin{aligned}
& \int_{-\pi}^{\pi}\left|\left(\sum_{k \in \mathbb{Z}}\left|\widehat{\varphi}_{n}(\xi+2 k \pi)\right|^{2}\right)^{\frac{1}{2}}-\left(\sum_{k \in \mathbb{Z}}|\widehat{\varphi}(\xi+2 k \pi)|^{2}\right)^{\frac{1}{2}}\right|^{2} d \xi \\
\leq & \int_{-\pi}^{\pi} \sum_{k \in \mathbb{Z}}\left|\left(\widehat{\varphi}_{n}-\widehat{\varphi}\right)(\xi+2 k \pi)\right|^{2} d \xi=\left\|\widehat{\varphi}_{n}-\widehat{\varphi}\right\|_{L^{2}(\mathbb{R})}^{2} \rightarrow 0, \quad \text { as } n \rightarrow \infty .
\end{aligned}
$$

Since $\sum_{k \in \mathbb{Z}}\left|\widehat{\varphi}_{n}(\xi+2 k \pi)\right|^{2}=1$, a.e. $\xi \in \mathbb{T}$, (i) holds.

To see (ii), consider the low-pass filters $m_{n}$ associated with $\varphi_{n}$. By continuity of $M^{-1}$ (as a mapping from $H^{\alpha}(\mathbb{R})$ into $H^{\alpha}(\mathbb{T})$ ) there exists a $2 \pi$-periodic function $m \in H^{\alpha}(\mathbb{T})$ such that $m_{n} \rightarrow m$ in $H^{\alpha}(\mathbb{T})$. Now, for each fixed $\xi \in \mathbb{R}$, the Sobolev Embedding Theorems (in $\mathbb{R}$ and $\mathbb{T}$ ) imply that

$$
\widehat{\varphi}(2 \xi)=\lim _{n \rightarrow \infty} \widehat{\varphi}_{n}(2 \xi)=\lim _{n \rightarrow \infty} \widehat{\varphi}_{n}(\xi) m_{n}(\xi)=\widehat{\varphi}(\xi) m(\xi) .
$$

This shows (ii) and establishes the lemma. 
Remark 3.10. It is well-known (see Chapter 3 of $[\mathbf{7}]$ ) that, for an $\alpha$-localized scaling function, with $\alpha>\frac{1}{2}$, the equality

$$
\sum_{k \in \mathbb{Z}}|\widehat{\varphi}(\xi+2 k \pi)|^{2}=1
$$

holds at every point $\xi \in[-\pi, \pi]$. In fact, whenever $\widehat{\varphi} \in H^{\alpha}(\mathbb{R})$, the series on the left of $(3.11)$ converges uniformly in $[-\pi, \pi]$ to a function in the periodic Sobolev space $H^{\alpha}(\mathbb{T})$.

The statement of Lemma 3.9 can also be phrased in the following way: $\mathcal{S}_{\alpha}$ is a complete metric space. Note that, in view of Example 2.5, $\mathcal{E}_{\alpha}$ is not complete and, consequently, the homeomorphism $M$, between $\mathcal{E}_{\alpha}$ and $\mathcal{S}_{\alpha}$, will preserve the topologies, but not the metrics! The following example illustrates this situation in a more clear way:

Example 3.12. $M$ is not continuous as a mapping from $\mathcal{F}_{\alpha}$ to $H^{\alpha}(\mathbb{R})$.

Let $\left\{\left(a_{n}, c_{n}\right)\right\}$ be a sequence of points in the punctured circle of Example 2.5 converging to $(1,1)$. Then, the functions $m_{n}=m_{\left(a_{n}, c_{n}\right)}$ belong to $\mathcal{E}_{\alpha}$ and converge in the $H^{\alpha}(\mathbb{T})$-topology to $m=m_{(1,1)}$ (which is not in $\mathcal{E}_{\alpha}$, but belongs to $\left.\mathcal{F}_{\alpha}\right)$. We claim that $\widehat{\varphi}_{n}=M\left(m_{n}\right)$ cannot converge in the $H^{\alpha}(\mathbb{R})$-topology to anything. Indeed, if it did, the limit point must be $\widehat{\varphi}=\prod_{j=1}^{\infty} m\left(2^{-j}\right.$.) (this is, for instance, a consequence of Lemma 2.10). But $\varphi=\frac{1}{3} \chi_{[-3,0]}$, which is not a scaling function and, therefore, contradicts Lemma 3.9.

These pathological behaviors illustrate some of the difficulties we will encounter when proving Theorem 3.8. We will not be able to avoid the use Cohen's condition, as we did in section 2. However, the following lemma contains most of the information needed.

Lemma 3.13. Let $\alpha>\frac{1}{2}$. Suppose that $m_{n} \rightarrow m$ in $\mathcal{E}_{\alpha}$ and let $\widehat{\varphi}_{n}=M\left(m_{n}\right), \widehat{\varphi}=M(m)$. Then

$$
\left\|\widehat{\varphi}_{n}-\widehat{\varphi}\right\|_{*}^{2} \equiv \sup _{\xi \in \mathbb{T}} \sum_{k \in \mathbb{Z}}\left|\widehat{\varphi}_{n}(\xi+2 k \pi)-\widehat{\varphi}(\xi+2 k \pi)\right|^{2} \rightarrow 0, \quad \text { as } n \rightarrow \infty \text {. }
$$

Proof: Since, by Cohen's Theorem, $\varphi_{n}, \varphi$ are scaling functions, it follows from Remark 3.10 that

$\sum_{k \in \mathbb{Z}}\left|\widehat{\varphi}_{n}(\xi+2 k \pi)\right|^{2}=\sum_{k \in \mathbb{Z}}|\widehat{\varphi}(\xi+2 k \pi)|^{2}=1, \quad$ for every $\xi \in[-\pi, \pi], n \geq 1$, 
where all the series converge uniformly on $[-\pi, \pi]$. Let us fix a positive number $\varepsilon \in(0,1)$. Then, we can find an integer $k_{0} \geq 1$ such that

$$
\sum_{|k| \geq k_{0}}|\widehat{\varphi}(\xi+2 k \pi)|^{2}<\varepsilon, \quad \text { for all } \xi \in[-\pi, \pi] .
$$

Consider now the compact set $K=\left[-\left(2 k_{0}+1\right) \pi,\left(2 k_{0}+1\right) \pi\right]$. Then, by Lemma 2.10, we can find a positive integer $n_{0}$ such that

$$
\left|\widehat{\varphi}_{n}(\eta)-\widehat{\varphi}(\eta)\right|<\frac{\varepsilon}{2 k_{0}+1}, \quad \text { for all } \eta \in K, n \geq n_{0} .
$$

Therefore, when $\xi \in[-\pi, \pi]$ and $n \geq n_{0}$ we have

$$
\begin{aligned}
& \sum_{k \in \mathbb{Z}}\left|\widehat{\varphi}_{n}(\xi+2 k \pi)-\widehat{\varphi}(\xi+2 k \pi)\right|^{2} \leq \sum_{|k| \leq k_{0}}\left|\left(\widehat{\varphi}_{n}-\widehat{\varphi}\right)(\xi+2 k \pi)\right|^{2} \\
+ & 2 \sum_{|k| \geq k_{0}+1}|\widehat{\varphi}(\xi+2 k \pi)|^{2}+2 \sum_{|k| \geq k_{0}+1}\left|\widehat{\varphi}_{n}(\xi+2 k \pi)\right|^{2}=I+I I+I I I .
\end{aligned}
$$

By (3.15) and (3.16), we easily see that

$$
I+I I<\sum_{|k| \leq k_{0}} \frac{\varepsilon^{2}}{\left(2 k_{0}+1\right)^{2}}+2 \varepsilon<3 \varepsilon .
$$

On the other hand,

$$
\begin{aligned}
\frac{1}{2} I I I= & 1-\sum_{|k| \leq k_{0}}\left|\widehat{\varphi}_{n}(\xi+2 k \pi)\right|^{2} \\
= & \sum_{|k| \geq k_{0}+1}|\widehat{\varphi}(\xi+2 k \pi)|^{2} \\
& +\left(\sum_{|k| \leq k_{0}}|\widehat{\varphi}(\xi+2 k \pi)|^{2}-\sum_{|k| \leq k_{0}}\left|\widehat{\varphi}_{n}(\xi+2 k \pi)\right|^{2}\right) \\
< & \varepsilon+2 \sum_{|k| \leq k_{0}}\left|\left(\widehat{\varphi}-\widehat{\varphi}_{n}\right)(\xi+2 k \pi)\right|<\varepsilon+2 \varepsilon=3 \varepsilon .
\end{aligned}
$$

Putting these two estimates together, we obtain that $I+I I+I I I<9 \varepsilon$ and, therefore,

$$
\sup _{\xi \in \mathbb{T}} \sum_{k \in \mathbb{Z}}\left|\widehat{\varphi}_{n}(\xi+2 k \pi)-\widehat{\varphi}(\xi+2 k \pi)\right|^{2} \rightarrow 0, \quad \text { as } n \rightarrow \infty,
$$

completing the proof of the lemma. 


\subsection{The proof of the theorem.}

At this point, the proof of Theorem 3.8 reduces to showing that $M$ is a continuous map from $\mathcal{E}_{\alpha}$ into the Sobolev space $H^{\alpha}(\mathbb{R})$. That is, if $m, m_{n} \in \mathcal{E}_{\alpha}, n=1,2, \ldots$, and $m_{n} \rightarrow m$ in $H^{\alpha}(\mathbb{T})$, then we need to show that $\widehat{\varphi}_{n} \rightarrow \widehat{\varphi}$ in the topology of $H^{\alpha}(\mathbb{R})$, where

$$
\widehat{\varphi}_{n}(\xi)=\prod_{j=1}^{\infty} m_{n}\left(2^{-j} \xi\right) \quad \text { and } \quad \widehat{\varphi}(\xi)=\prod_{j=1}^{\infty} m\left(2^{-j} \xi\right), \quad \xi \in \mathbb{R} .
$$

To do this, we use the standard definition of norm in $H^{\alpha}(\mathbb{R})$ in terms of distributional derivatives and $L^{2}$-modulus of continuity. That is, when $\alpha=k+\varepsilon$, where $k \in \mathbb{Z}^{+} \cup\{0\}$ and $0 \leq \varepsilon<1$, a function $g$ belongs to $H^{\alpha}(\mathbb{R})$ if and only if $g, \ldots, g^{(k)} \in L^{2}(\mathbb{R})$ and

$$
\omega_{\varepsilon}\left(g^{(k)}\right)=\left[\int_{\mathbb{R}} \int_{\mathbb{R}}\left|g^{(k)}(x+h)-g^{(k)}(x)\right|^{2} d x \frac{d h}{|h|^{1+2 \varepsilon}}\right]^{\frac{1}{2}}<\infty
$$

(we let $\omega_{\varepsilon} \equiv 0$ when $\varepsilon=0$ ), having: $\|g\|_{H^{\alpha}(\mathbb{R})} \simeq\|g\|_{L^{2}(\mathbb{R})}+\left\|g^{(k)}\right\|_{L^{2}(\mathbb{R})}+$ $\omega_{\varepsilon}\left(g^{(k)}\right)$.

We use similar norm definitions in the periodic Sobolev spaces $H^{\alpha}(\mathbb{T})$, after replacing the integrals over $\mathbb{R}$, by integrals over $\mathbb{T}$.

Starting with the proof of the theorem, first of all note that, by Lemma 3.13 and the Dominated Convergence Theorem:

$$
\lim _{n \rightarrow \infty}\left\|\widehat{\varphi}_{n}-\widehat{\varphi}\right\|_{L^{2}(\mathbb{R})}^{2}=\lim _{n \rightarrow \infty} \int_{\mathbb{T}} \sum_{k \in \mathbb{Z}}\left|\left(\widehat{\varphi}_{n}-\widehat{\varphi}\right)(\xi+2 k \pi)\right|^{2} d \xi=0 .
$$

To show that $\left\|\widehat{\varphi}_{n}-\widehat{\varphi}\right\|_{H^{\alpha}(\mathbb{R})} \rightarrow 0$, as $n \rightarrow \infty$, we split the proof into different cases, according to the value of $\alpha$.

Case 1: $\frac{1}{2}<\alpha<1$.

It suffices to see that

(3.18) $\omega_{\alpha}\left(\widehat{\varphi}_{n}-\widehat{\varphi}\right)$

$$
=\left[\int_{\mathbb{R}} \int_{\mathbb{R}}\left|\left(\widehat{\varphi}_{n}-\widehat{\varphi}\right)(\xi)-\left(\widehat{\varphi}_{n}-\widehat{\varphi}\right)(\xi+\eta)\right|^{2} \frac{d \xi d \eta}{|\eta|^{1+2 \alpha}}\right]^{\frac{1}{2}} \rightarrow 0, \quad \text { as } n \rightarrow \infty .
$$

In the same way as in (2.11) we can write, for $\xi, \eta \in \mathbb{R}$ :

$$
\begin{aligned}
& \widehat{\varphi}(\xi)-\widehat{\varphi}(\xi+\eta) \\
& =\sum_{L=1}^{\infty} \prod_{j=1}^{L-1} m\left(2^{-j}(\xi+\eta)\right)\left[m\left(2^{-L} \xi\right)-m\left(2^{-L}(\xi+\eta)\right)\right] \widehat{\varphi}\left(2^{-L} \xi\right),
\end{aligned}
$$


where the series converges absolutely. Using (3.19) with $\widehat{\varphi}_{n}$ and $\widehat{\varphi}$, and subtracting, we obtain

$$
\begin{aligned}
& \left(\widehat{\varphi}_{n}-\widehat{\varphi}\right)(\xi)-\left(\widehat{\varphi}_{n}-\widehat{\varphi}\right)(\xi+\eta) \\
& =\sum_{L=1}^{\infty}\left\{\sum_{h=1}^{L-1 h-1} \prod_{j=1} m\left(2^{-j}(\xi+\eta)\right)\left[\left(m_{n}-m\right)\left(2^{-h}(\xi+\eta)\right)\right] \prod_{j=h+1}^{L-1} m_{n}\left(2^{-j}(\xi+\eta)\right)\right. \\
& \left.\quad \times\left[m_{n}\left(2^{-L} \xi\right)-m_{n}\left(2^{-L}(\xi+\eta)\right)\right] \widehat{\varphi}_{n}\left(2^{-L} \xi\right)\right\} \\
& +\sum_{L=1}^{\infty} \prod_{j=1}^{L-1} m\left(2^{-j}(\xi+\eta)\right)\left[\left(m_{n}-m\right)\left(2^{-L} \xi\right)-\left(m_{n}-m\right)\left(2^{-L}(\xi+\eta)\right)\right] \widehat{\varphi}_{n}\left(2^{-L} \xi\right) \\
& +\sum_{L=1}^{\infty} \prod_{j=1}^{L-1} m\left(2^{-j}(\xi+\eta)\right)\left[m\left(2^{-L} \xi\right)-m\left(2^{-L}(\xi+\eta)\right)\right]\left[\left(\widehat{\varphi}_{n}-\widehat{\varphi}\right)\left(2^{-L} \xi\right)\right] \\
& =A+B+C .
\end{aligned}
$$

It is enough to show that each of the terms $\omega_{\alpha}(A), \omega_{\alpha}(B)$ and $\omega_{\alpha}(C)$ tends to 0 , as $n \rightarrow \infty$. We start with $A$. Estimating the first three multiplicative blocks in $A$ with the $\|\cdot\|_{\infty}$-norm, and changing variables in $\xi$ and $\eta$, we obtain

$$
\begin{aligned}
& \omega_{\alpha}(A) \leq\left\|m_{n}-m\right\|_{\infty} \sum_{L=1}^{\infty} \sum_{h=1}^{L-1} 2^{-L\left(\alpha-\frac{1}{2}\right)} \\
& \times {\left[\int_{\mathbb{R}} \int_{\mathbb{R}}\left|m_{n}(\xi)-m_{n}(\xi+\eta)\right|^{2}\left|\widehat{\varphi}_{n}(\xi)\right|^{2} \frac{d \xi d \eta}{|\eta|^{1+2 \alpha}}\right]^{\frac{1}{2}} } \\
& \leq C\left\|m_{n}-m\right\|_{H^{\alpha}(\mathbb{T})}\left\|\widehat{\varphi}_{n}\right\|_{*}\left[\int_{\mathbb{R}} \int_{\mathbb{T}}\left|m_{n}(\xi)-m_{n}(\xi+\eta)\right|^{2} \frac{d \xi d \eta}{|\eta|^{1+2 \alpha}}\right]^{\frac{1}{2}}
\end{aligned}
$$

where in the second inequality we have periodized the integral in $\xi$ and used the Sobolev Embedding Theorem. Now, it follows from Lemma 3.13 that $\left\|\widehat{\varphi}_{n}\right\|_{*}$ is uniformly bounded and, therefore,

$$
\omega_{\alpha}(A) \leq C\left\|m_{n}-m\right\|_{H^{\alpha}(\mathbb{T})} \rightarrow 0, \quad \text { as } n \rightarrow \infty .
$$


One deals similarly with $B$, obtaining:

$$
\begin{aligned}
\omega_{\alpha}(B) \leq \sum_{L=1}^{\infty} 2^{-L\left(\alpha-\frac{1}{2}\right)}\left\|\widehat{\varphi}_{n}\right\|_{*} & {\left[\int_{\mathbb{T}} \int_{\mathbb{T}}\left|m_{n}(\xi)-m_{n}(\xi+\eta)\right|^{2} \frac{d \xi d \eta}{|\eta|^{1+2 \alpha}}\right.} \\
& \left.+8 \pi \int_{|\eta|>\pi}\left\|m_{n}-m\right\|_{\infty}^{2} \frac{d \eta}{|\eta|^{1+2 \alpha}}\right]^{\frac{1}{2}} \\
\leq C\left\|m_{n}-m\right\|_{H^{\alpha}(\mathbb{T})} \rightarrow & 0, \quad \text { as } n \rightarrow \infty .
\end{aligned}
$$

Finally, the third term is bounded by

$$
\begin{aligned}
\omega_{\alpha}(C) & \leq \sum_{L=1}^{\infty} 2^{-L\left(\alpha-\frac{1}{2}\right)}\left\|\widehat{\varphi}_{n}-\widehat{\varphi}\right\|_{*}\left[\int_{\mathbb{R}} \int_{\mathbb{T}}\left|m_{n}(\xi)-m_{n}(\xi+\eta)\right|^{2} \frac{d \xi d \eta}{|\eta|^{1+2 \alpha}}\right]^{\frac{1}{2}} \\
& \leq C\left\|\widehat{\varphi}_{n}-\widehat{\varphi}\right\|_{*} \rightarrow 0, \quad \text { as } n \rightarrow \infty
\end{aligned}
$$

where the convergence to 0 follows from Lemma 3.13. This shows (3.18) and, together with (3.17), completes the proof of case 1.

Case 2: $\alpha=k \in \mathbb{Z}^{+}$.

We need to show that:

$$
\left\|D^{(k)} \widehat{\varphi}_{n}-D^{(k)} \widehat{\varphi}\right\|_{L^{2}(\mathbb{R})} \rightarrow 0, \quad \text { as } n \rightarrow \infty .
$$

Recall that $D^{(k)} \widehat{\varphi}$ is the distributional derivative of an infinite product of periodic functions. Note that, when $0 \leq h \leq k-1$, the distribution $m^{(h)}$ may be considered as $2 \pi$-periodic function in $C^{k-h-1}(\mathbb{R})$, while $m^{(k)}$ is (a.e.) a function in $L^{2}(\mathbb{T})$. Moreover, the following formula, appearing in Chapter 4 of [7], holds (here $\delta_{j}^{\ell}$ denotes the usual Krönecker symbol):

Lemma 3.21. Let $k \in \mathbb{Z}^{+}$. If $m \in H^{k}(\mathbb{T})$, with $m(0)=1$, $|m| \leq 1$, and $\widehat{\varphi}$ is defined by the infinite product formula (3.6) then, the $k^{\text {th }}$ distributional derivative of $\widehat{\varphi}$ can be written as:

$$
D^{(k)} \widehat{\varphi}(\xi)=\sum_{L=1}^{\infty} \sum_{\substack{\ell \in\left(\mathbb{Z}^{+}\right)^{k} \\ \sup _{1 \leq i \leq k} \ell_{i}=L}}\left(\frac{1}{2}\right)^{|\ell|} \prod_{j=1}^{L} m^{\left(\varepsilon_{j}^{\ell}\right)}\left(2^{-j} \xi\right) \widehat{\varphi}\left(2^{-L} \xi\right)
$$

where $|\ell|=\ell_{1}+\cdots+\ell_{k}, \varepsilon_{j}^{\ell}=\sum_{i=1}^{k} \delta_{j}^{\ell_{i}}, \ell=\left(\ell_{1}, \ldots, \ell_{k}\right) \in\left(\mathbb{Z}^{+}\right)^{k}, j \geq 1$, and the series in (3.22) converges absolutely for a.e. $\xi \in \mathbb{R}$. 
The notation in (3.22) can be interpreted in the following way: for a fixed $L \geq 1$ and a given multi-index $\ell=\left(\ell_{1}, \ldots, \ell_{k}\right)$ we spread the $k$ derivatives among the $L$ first terms of the infinite product, where each $\ell_{i}$ indicates that the $\ell_{i}^{\text {th }}$-term is differentiated one time. The condition $\sup _{1<i<k} \ell_{i}=L$ guarantees that the $L^{\text {th }}$-term is always considered and, therefore, that there are no repetitions in the process. Note that, when $\ell=(L, \ldots, L)$, all the derivatives are concentrated in one term and, therefore, the function $m^{(k)}$ (which is in $L^{2}(\mathbb{T}$ ), but may not be continuous) comes into play. This will give rise to different cases when proving (3.20) above.

Using (3.22), we can write:

$$
\begin{aligned}
& D^{(k)} \widehat{\varphi}_{n}(\xi)-D^{(k)} \widehat{\varphi}(\xi) \\
& =\sum_{L=1}^{\infty}\left\{\sum _ { \substack { \operatorname { s u p } _ { \ell , L } \ell _ { i } = L \\
\ell \neq ( L , \ldots , L ) } } 2 ^ { - | \ell | } \left[\sum_{h=1}^{L} \prod_{j=1}^{h-1} m^{\left(\varepsilon_{j}^{\ell}\right)}\left(2^{-j} \xi\right)\left[\left(m_{n}-m\right)^{\left(\varepsilon_{h}^{\ell}\right)}\left(2^{-h} \xi\right)\right]\right.\right. \\
& \times \prod_{j=h+1}^{L} m_{n}{ }^{\left(\varepsilon_{j}^{\ell}\right)}\left(2^{-j} \xi\right) \widehat{\varphi}_{n}\left(2^{-L} \xi\right) \\
& \left.+\prod_{j=1}^{L} m^{\left(\varepsilon_{j}^{\ell}\right)}\left(2^{-j} \xi\right)\left[\widehat{\varphi}_{n}\left(2^{-L} \xi\right)-\widehat{\varphi}\left(2^{-L} \xi\right)\right]\right] \\
& +\frac{1}{2^{k L}}\left[\sum_{h=1}^{L-1} \prod_{j=1}^{h-1} m\left(2^{-j} \xi\right)\left[\left(m_{n}-m\right)\left(2^{-h} \xi\right)\right]\right. \\
& \times \prod_{j=h+1}^{L-1} m_{n}\left(2^{-j} \xi\right) m_{n}{ }^{(k)}\left(2^{-L} \xi\right) \widehat{\varphi}_{n}\left(2^{-L} \xi\right) \\
& +\prod_{j=1}^{L-1} m\left(2^{-j} \xi\right)\left[\left(m_{n}-m\right)^{(k)}\left(2^{-L} \xi\right)\right] \widehat{\varphi}_{n}\left(2^{-L} \xi\right) \\
& \left.\left.+\prod_{j=1}^{L-1} m\left(2^{-j} \xi\right) m^{(k)}\left(2^{-L} \xi\right)\left[\left(\widehat{\varphi}_{n}-\widehat{\varphi}\right)\left(2^{-L} \xi\right)\right]\right]\right\} \\
& =\{[I+I I]+[I I I+I V+V]\} .
\end{aligned}
$$


An upper bound for the $L^{2}(\mathbb{R})$-norm of the first two terms can be obtained easily, estimating the periodic functions with $\|\cdot\|_{\infty}$, and changing variables:

$$
\begin{gathered}
\|I\|_{L^{2}(\mathbb{R})} \leq \sum_{L=1}^{\infty} \sum_{\substack{\sup _{\ell \neq(L, \ldots, L)} \ell_{i}=L \\
2^{L}}} \sum_{h=1}^{L} 2^{L / 2} \prod_{j=1}^{h-1}\left\|m^{\left(\varepsilon_{j}^{\ell}\right)}\right\|_{\infty}\left\|\left(m_{n}-m\right)^{\left(\varepsilon_{h}^{\ell}\right)}\right\|_{\infty} \\
\times \prod_{j=h+1}^{L}\left\|m_{n}^{\left(\varepsilon_{j}^{\ell}\right)}\right\|_{\infty}\left\|\widehat{\varphi}_{n}\right\|_{L^{2}(\mathbb{R})} \\
\leq C\|m\|_{H^{\alpha}(\mathbb{T})}^{k}\left\|m_{n}-m\right\|_{H^{\alpha}(\mathbb{T})}\left\|m_{n}\right\|_{H^{\alpha}(\mathbb{T})}^{k} \sum_{L=1}^{\infty} \frac{L^{k+1}}{2^{L / 2}} \\
\leq C\left\|m_{n}-m\right\|_{H^{\alpha}(\mathbb{T})} \rightarrow 0, \quad \text { as } n \rightarrow \infty,
\end{gathered}
$$

and

$$
\|I I\|_{L^{2}(\mathbb{R})} \leq \sum_{L=1}^{\infty} \frac{L^{k+1}}{2^{L / 2}}\|m\|_{H^{\alpha}(\mathbb{T})}^{k}\left\|\widehat{\varphi}_{n}-\widehat{\varphi}\right\|_{L^{2}(\mathbb{R})} \rightarrow 0, \quad \text { as } n \rightarrow \infty
$$

where the convergence to 0 in the second case follows from (3.17). For the other three terms, we cannot take the $\|\cdot\|_{\infty}$-norm of $m^{(k)}$, and need to periodize the integral, obtaining:

$$
\begin{aligned}
\|I I I\|_{L^{2}(\mathbb{R})} & \leq \sum_{L=1}^{\infty} \frac{1}{2^{k L}} \sum_{h=1}^{L-1} 2^{L / 2}\left\|m_{n}-m\right\|_{\infty}\left\|\widehat{\varphi}_{n}\right\|_{*}\left\|m_{n}{ }^{(k)}\right\|_{L^{2}(\mathbb{T})} \\
& \leq C\left\|m_{n}-m\right\|_{H^{\alpha}(\mathbb{T})} \rightarrow 0, \quad \text { as } n \rightarrow \infty \\
\|I V\|_{L^{2}(\mathbb{R})} & \leq \sum_{L=1}^{\infty} \frac{1}{2^{k L}} 2^{L / 2}\left\|\widehat{\varphi}_{n}\right\|_{*}\left\|m_{n}{ }^{(k)}-m^{(k)}\right\|_{L^{2}(\mathbb{T})} \\
& \leq C\left\|m_{n}-m\right\|_{H^{\alpha}(\mathbb{T})} \rightarrow 0, \quad \text { as } n \rightarrow \infty
\end{aligned}
$$


and

$$
\|V\|_{L^{2}(\mathbb{R})} \leq \sum_{L=1}^{\infty} \frac{1}{2^{k L}} 2^{L / 2}\left\|\widehat{\varphi}_{n}-\widehat{\varphi}\right\|_{*}\left\|m^{(k)}\right\|_{L^{2}(\mathbb{T})} \rightarrow 0, \quad \text { as } n \rightarrow \infty
$$

where in the last limit we use Lemma 3.13. This shows (3.20) and completes the proof of case 2 .

Case 3: $\alpha=k+\varepsilon, \quad k \in \mathbb{Z}^{+}, 0<\varepsilon<1$.

This case is a little more tedious to write, mainly because of the cumbersome notation needed; however, all the estimations follow from the same type of arguments we used previously. We only present here those that require major modifications, leaving the others to the reader (or referring to section 3.3 of [3] for further details). We have to show that

$(3.23) \omega_{\varepsilon}\left(D^{(k)} \widehat{\varphi}_{n}-D^{(k)} \widehat{\varphi}\right)$

$$
=\left[\int_{\mathbb{R}} \int_{\mathbb{R}}\left|D^{(k)}\left(\widehat{\varphi}_{n}-\widehat{\varphi}\right)(\xi)-D^{(k)}\left(\widehat{\varphi}_{n}-\widehat{\varphi}\right)(\xi+\eta)\right|^{2} \frac{d \xi d \eta}{|\eta|^{1+2 \varepsilon}}\right]^{\frac{1}{2}} \rightarrow 0, \text { as } n \rightarrow \infty .
$$

By using (3.22), we obtain a similar decomposition to the one in (3.19), this time involving derivatives:

$$
\begin{aligned}
& D^{(k)} \widehat{\varphi}(\xi)-D^{(k)} \widehat{\varphi}(\xi+\eta) \\
& =\sum_{\substack { L=1 \\
\begin{subarray}{c}{\ell \in\left(\mathbb{Z}^{+}\right)^{k} \\
\sup \ell_{i}=L{ L = 1 \\
\begin{subarray} { c } { \ell \in ( \mathbb { Z } ^ { + } ) ^ { k } \\
\operatorname { s u p } \ell _ { i } = L } }\end{subarray}}^{\infty} \sum^{|\ell|} \sum_{p=1}^{L}\left\{\prod_{j=1}^{p-1} m^{\left(\varepsilon_{j}^{\ell}\right)}\left(2^{-j}(\xi+\eta)\right)\left[m^{\left(\varepsilon_{p}^{\ell}\right)}\left(2^{-p} \xi\right)-m^{\left(\varepsilon_{p}^{\ell}\right)}\left(2^{-p}(\xi+\eta)\right)\right]\right. \\
& \left.\times \prod_{j=p+1}^{L} m^{\left(\varepsilon_{j}^{\ell}\right)}\left(2^{-j} \xi\right) \widehat{\varphi}\left(2^{-L} \xi\right)\right\} \\
& \left.+\prod_{j=1}^{L} m^{\left(\varepsilon_{j}^{\ell}\right)}\left(2^{-j}(\xi+\eta)\right)\left[\widehat{\varphi}\left(2^{-L} \xi\right)-\widehat{\varphi}\left(2^{-L}(\xi+\eta)\right)\right]\right]
\end{aligned}
$$


To simplify the notation, we will write $\left(\Delta_{\eta} F\right)(\xi) \equiv F(\xi)-F(\xi+\eta)$. Now, using the previous equality with $\widehat{\varphi}$ and $\widehat{\varphi}_{n}$, and subtracting both quantities, we obtain

$$
\begin{aligned}
& D^{(k)}\left(\widehat{\varphi}_{n}-\widehat{\varphi}\right)(\xi)-D^{(k)}\left(\widehat{\varphi}_{n}-\widehat{\varphi}\right)(\xi+\eta) \\
& =\sum_{L=1}^{\infty} \sum_{\sup \ell_{i}=L} \frac{1}{2^{|\ell|}}\left\{\sum _ { p = 1 } ^ { L } \left[\sum _ { q = 1 } ^ { p - 1 } \left\{\prod_{j=1}^{q-1} m^{\left(\varepsilon_{j}^{\ell}\right)}\left(2^{-j}(\xi+\eta)\right)\left[\left(m_{n}-m\right)^{\left(\varepsilon_{q}^{\ell}\right)}\left(2^{-q}(\xi+\eta)\right)\right]\right.\right.\right. \\
& \left.\times \prod_{j=q+1}^{p-1} m_{n}^{\left(\varepsilon_{j}^{\ell}\right)}\left(2^{-j}(\xi+\eta)\right)\left[\left(\Delta_{\eta} m_{n}{ }^{\left(\varepsilon_{p}^{\ell}\right)}\left(2^{-p} \cdot\right)\right)(\xi)\right] \prod_{j=p+1}^{L} m_{n}{ }^{\left(\varepsilon_{j}^{\ell}\right)}\left(2^{-j} \xi\right) \widehat{\varphi}_{n}\left(2^{-L} \xi\right)\right\} \\
& +\prod_{j=1}^{p-1} m^{\left(\varepsilon_{j}^{\ell}\right)}\left(2^{-j}(\xi+\eta)\right)\left[\left(\Delta_{\eta}\left(m_{n}-m\right)^{\left(\varepsilon_{p}^{\ell}\right)}\left(2^{-p} \cdot\right)\right)(\xi)\right] \prod_{j=p+1}^{L} m_{n}{ }^{\left(\varepsilon_{j}^{\ell}\right)}\left(2^{-j} \xi\right) \widehat{\varphi}_{n}\left(2^{-L_{\xi}}\right) \\
& +\sum_{q=p+1}^{L}\left\{\prod_{j=1}^{p-1} m^{\left(\varepsilon_{j}^{\ell}\right)}\left(2^{-j}(\xi+\eta)\right)\left[\left(\Delta_{\eta} m^{\left(\varepsilon_{p}^{\ell}\right)}\left(2^{-p} \cdot\right)\right)(\xi)\right] \prod_{j=p+1}^{q-1} m^{\left(\varepsilon_{j}^{\ell}\right)}\left(2^{-j} \xi\right)\right. \\
& \left.\times\left[\left(m_{n}-m\right)^{\left(\varepsilon_{q}^{\ell}\right)}\left(2^{-q} \xi\right)\right] \prod_{j=q+1}^{L} m_{n}^{\left(\varepsilon_{j}^{\ell}\right)}\left(2^{-j} \xi\right) \widehat{\varphi}_{n}\left(2^{-L} \xi\right)\right\} \\
& \left.+\prod_{j=1}^{p-1} m^{\left(\varepsilon_{j}^{\ell}\right)}\left(2^{-j}(\xi+\eta)\right)\left[\left(\Delta_{\eta} m^{\left(\varepsilon_{p}^{\ell}\right)}\left(2^{-p} \cdot\right)\right)(\xi)\right] \prod_{j=p+1}^{L} m^{\left(\varepsilon_{j}^{\ell}\right)}\left(2^{-j} \xi\right)\left(\widehat{\varphi}_{n}-\widehat{\varphi}\right)\left(2^{-L} \xi\right)\right] \\
& +\sum_{q=1}^{L}\left[\prod_{j=1}^{q-1} m^{\left(\varepsilon_{j}^{\ell}\right)}\left(2^{-j}(\xi+\eta)\right)\left[\left(m_{n}-m\right)^{\left(\varepsilon_{q}^{\ell}\right)}\left(2^{-q}(\xi+\eta)\right)\right] \prod_{j=q+1}^{L} m_{n}{ }^{\left(\varepsilon_{j}^{\ell}\right)}\left(2^{-j}(\xi+\eta)\right)\right. \\
& \left.\times\left[\left(\Delta_{\eta} \widehat{\varphi}_{n}\left(2^{-L} \cdot\right)\right)(\xi)\right]\right] \\
& \left.+\prod_{j=1}^{L} m^{\left(\varepsilon_{j}^{\ell}\right)}\left(2^{-j}(\xi+\eta)\right)\left[\left(\Delta_{\eta}\left(\widehat{\varphi}_{n}-\widehat{\varphi}\right)\left(2^{-L} \cdot\right)\right)(\xi)\right]\right\} \\
& =\left[\sum_{L=1}^{\infty} \sum_{\boldsymbol{\ell} \neq(L, \ldots, L)}\right]+\left[\sum_{L=1}^{\infty} \sum_{\boldsymbol{\ell}=(L, \ldots, L)}\right] \\
& =\left[A_{1}+\cdots+A_{6}\right]+\left[B_{1}+\cdots+B_{6}\right]=A+B .
\end{aligned}
$$


The estimates needed to show that $\omega_{\varepsilon}(A) \rightarrow 0$, when $n \rightarrow \infty$, are essentially the same as in the previous cases: we isolate the factor that contains the difference $\left\|m_{n}-m\right\|_{\infty}$ (or, more generally, $\left\|m_{n}-m\right\|_{H^{\alpha}(\mathbb{T})}$ ), while we control the rest of the integral $\int_{\mathbb{R}} \int_{\mathbb{R}} \ldots \frac{d \xi d \eta}{|\eta|^{1+2 \varepsilon}}$ by periodizing in $\xi$, and with the increments $\Delta_{\eta} G$, for different choices of $G=m_{n}{ }^{\left(\varepsilon_{j}^{\ell}\right)}, m^{\left(\varepsilon_{j}^{\ell}\right)}, \widehat{\varphi}_{n}, \ldots$ To control the "tales of the products", one can use the following lemma, whose simple proof can be found in Chapter 4 of [7].

Lemma 3.24. Let $\mathcal{H}$ be the normed space (modulo null-functions) defined by

$\mathcal{H}=\left\{f: \mathbb{R} \rightarrow \mathbb{C}\right.$ measurable: $\left.\|f\|_{*}^{2}=\operatorname{ess-sup}_{\xi \in \mathbb{R}} \sum_{k \in \mathbb{Z}}|f(\xi+2 k \pi)|^{2}<\infty\right\}$.

Let $\tau$ be a $2 \pi$-periodic measurable function and let $P=P_{\tau}$ be the operator defined at $f \in \mathcal{H}$ by

$$
(P f)(\xi)=\tau(\xi / 2) f(\xi / 2), \quad \xi \in \mathbb{R} .
$$

Then, for every $f \in \mathcal{H}$

$$
\|P f\|_{*} \leq \operatorname{ess} \sup _{\xi \in \mathbb{T}}\left[|\tau(\xi)|^{2}+|\tau(\xi+\pi)|^{2}\right]^{\frac{1}{2}}\|f\|_{*} .
$$

After a change of variable, and periodization, the case $A_{1}$ becomes:

$$
\begin{gathered}
\omega_{\varepsilon}\left(A_{1}\right) \leq \sum_{L=1}^{\infty} \frac{L^{k}}{2^{L}} \sum_{p=1}^{L}(p-1)\|m\|_{\alpha}^{k}\left\|m_{n}-m\right\|_{\alpha}\left\|m_{n}\right\|_{\alpha}^{k} 2^{-p\left(\varepsilon-\frac{1}{2}\right)} \underset{\left.m_{n} P_{p+1}^{\ell}\right)}{P_{m_{n}} \ldots P_{\left(\varepsilon_{L}^{\ell}\right)} \widehat{\varphi}_{n} \|_{*}} \\
\times\left[\int_{\mathbb{R}} \int_{\mathbb{T}}\left|\left(\Delta_{\eta} m_{n}^{\left(\varepsilon_{p}^{\ell}\right)}\right)(\xi)\right|^{2} \frac{d \eta}{|\eta|^{1+2 \varepsilon}}\right]^{\frac{1}{2}} \\
\leq C \sum_{L=1}^{\infty} \frac{L^{k+2}}{2^{L / 2}} 2^{k / 2}\left\|\widehat{\varphi}_{n}\right\|_{*}\left\|m_{n}-m\right\|_{\alpha} \rightarrow 0, \quad \text { as } n \rightarrow \infty .
\end{gathered}
$$

One deals similarly with $A_{2}, A_{3}$ and $A_{5}$. In the case $A_{4}$, we use Lemma 3.13 to obtain

$$
\omega_{\varepsilon}\left(A_{4}\right) \leq C\left\|\widehat{\varphi}_{n}-\widehat{\varphi}\right\|_{*} \rightarrow 0, \quad \text { as } n \rightarrow \infty,
$$

while for $A_{6}$ we have

$$
\omega_{\varepsilon}\left(A_{6}\right) \leq C \omega_{\varepsilon}\left(\widehat{\varphi}_{n}-\widehat{\varphi}\right) \rightarrow 0, \quad \text { as } n \rightarrow \infty,
$$

the convergence to 0 following now from (3.18) in case 1 above. 
The calculations for $\omega_{\varepsilon}(B) \rightarrow 0$ follow the same pattern, the main difference with $A$ being that the $k^{\text {th }}$ derivatives have to be treated with $\|\cdot\|_{L^{2}(\mathbb{T})}$-norms rather than $\|\cdot\|_{\infty}$. The cases $B_{1}, B_{5}$, and $B_{6}$ contain the essential features of these modifications, and are presented below. The remaining cases can be easily derived from these.

When dealing with $B_{1}$, we are led to the following equality, in which we have separated the terms $1 \leq p \leq L-1$ and $p=L$ :

$$
\begin{aligned}
\omega_{\varepsilon}\left(B_{1}\right) \leq & \sum_{L=1}^{\infty} \frac{1}{2^{L}} \sum_{p=1}^{L-1}(p-1)\left\|m_{n}-m\right\|_{\infty} 2^{-p\left(\varepsilon-\frac{1}{2}\right)} \\
& \times\left[\int_{\mathbb{R}} \int_{\mathbb{R}}\left|\left(\Delta_{\eta} m_{n}\right)(\xi)\right|^{2}\left|m_{n}{ }^{(k)}\left(2^{-L+p} \xi\right) \widehat{\varphi}_{n}\left(2^{-L+p} \xi\right)\right|^{2} \frac{d \xi d \eta}{|\eta|^{1+2 \varepsilon}}\right]^{\frac{1}{2}} \\
& +\sum_{L=1}^{\infty} \frac{L-1}{2^{L}}\left\|m_{n}-m\right\|_{\infty} 2^{-L\left(\varepsilon-\frac{1}{2}\right)}\left\|\widehat{\varphi}_{n}\right\|_{*} \\
& \times\left[\int_{\mathbb{R}} \int_{\mathbb{T}}\left|\left(\Delta_{\eta} m_{n}^{(k)}\right)(\xi)\right|^{2} \frac{d \eta}{|\eta|^{1+2 \varepsilon}}\right]^{\frac{1}{2}} .
\end{aligned}
$$

The second summand is easily treated. For the first, we use the Sobolev Embedding Theorem to choose a $\theta \in\left(0, \frac{1}{2}\right)$ such that $0<\varepsilon+\theta<1$, and

$$
\left|m_{n}(\xi)-m_{n}(\xi+\eta)\right| \leq C\left\|m_{n}\right\|_{\alpha}|\eta|^{\varepsilon+\theta}, \quad \xi \in \mathbb{R},|\eta| \leq 1 .
$$

Then the double integral in the second line of the previous inequality can be estimated by:

$$
\begin{aligned}
& \int_{\mathbb{R}} \int_{\mathbb{R}}\left|\left(\Delta_{\eta} m_{n}\right)(\xi)\right|^{2}\left|m_{n}{ }^{(k)}\left(2^{-L+p} \xi\right) \widehat{\varphi}_{n}\left(2^{-L+p} \xi\right)\right|^{2} \frac{d \xi d \eta}{|\eta|^{1+2 \varepsilon}} \\
& \quad \leq C 2^{L-p}\left[\int_{\mathbb{R}}\left|m_{n}{ }^{(k)}(\xi)\right|^{2}\left|\widehat{\varphi}_{n}(\xi)\right|^{2} d \xi\right]\left[\int_{|\eta|>1} 4 \frac{d \eta}{|\eta|^{1+2 \varepsilon}}+\int_{|\eta| \leq 1} \frac{d \eta}{|\eta|^{1-2 \theta}}\right] \\
& \quad \leq C 2^{L-p}\left\|\widehat{\varphi}_{n}\right\|_{*}^{2}\left\|m_{n}{ }^{(k)}\right\|_{L^{2}(\mathbb{T})}^{2} .
\end{aligned}
$$

Thus,

$$
\omega_{\varepsilon}\left(B_{1}\right) \leq C \sum_{L=1}^{\infty} \frac{L^{2}}{2^{L / 2}}\left\|m_{n}-m\right\|_{\infty}+C \sum_{L=1}^{\infty} \frac{L}{2^{L / 2}}\left\|m_{n}-m\right\|_{\infty} \rightarrow 0
$$

as $n$ approaches $\infty$. 
To deal with $B_{5}$, we use a Lipschitz condition that is a little stronger than (3.25):

$$
\left\|\widehat{\varphi}(\cdot)-\widehat{\varphi}_{n}(\cdot+\eta)\right\|_{*} \leq \begin{cases}C|\eta|^{\frac{1}{2}}\left\|\widehat{\varphi}_{n}\right\|_{H^{1}(\mathbb{R})}, & \text { when } 0<\varepsilon<\frac{1}{2} \\ C|\eta|^{\gamma}\left\|\widehat{\varphi}_{n}\right\|_{H^{\frac{1}{2}+\gamma}(\mathbb{R})}, & \text { when } \frac{1}{2} \leq \varepsilon<1,\end{cases}
$$

for a suitable $\gamma \in(\varepsilon, 1)$ (see, e.g., Chapter 4 of [7]). Now, no matter what $\varepsilon$ is, we have

$$
\begin{aligned}
\omega_{\varepsilon}\left(B_{5}\right) \leq & \sum_{L=1}^{\infty} \frac{1}{2^{L}} \sum_{q=1}^{L-1} 2^{-L\left(\varepsilon-\frac{1}{2}\right)}\left\|m_{n}-m\right\|_{\infty} \\
& \times\left[\int_{\mathbb{R}} \int_{\mathbb{R}}\left|m_{n}{ }^{(k)}(\xi)\right|^{2}\left|\left(\Delta_{\eta} \widehat{\varphi}_{n}\right)(\xi)\right|^{2} \frac{d \xi d \eta}{|\eta|^{1+2 \varepsilon}}\right]^{\frac{1}{2}} \\
& +\sum_{L=1}^{\infty} \frac{1}{2^{L}} 2^{-L\left(\varepsilon-\frac{1}{2}\right)}\left[\int_{\mathbb{R}} \int_{\mathbb{R}}\left|\left(m_{n}-m\right)^{(k)}(\xi)\right|^{2}\left|\left(\Delta_{\eta} \widehat{\varphi}_{n}\right)(\xi)\right|^{2} \frac{d \xi d \eta}{|\eta|^{1+2 \varepsilon}}\right]^{\frac{1}{2}}
\end{aligned}
$$

Separating the integrals over $\eta$ in two parts: $\int_{|\eta| \leq 1}+\int_{|\eta|>1}$, using (3.26), and periodizing in $\xi$, we obtain:

$$
\omega_{\varepsilon}\left(B_{5}\right) \leq C\left\|m_{n}-m\right\|_{H^{\alpha}(\mathbb{T})} \rightarrow 0, \quad \text { as } n \rightarrow \infty .
$$

Finally, for $B_{6}$, we replace $\widehat{\varphi}$ by $\widehat{\varphi}_{n}-\widehat{\varphi}$ in the Lipschitz condition (3.26), and use the same argument as for $B_{5}$, obtaining, when $0<\varepsilon<\frac{1}{2}$,

$$
\omega_{\varepsilon}\left(B_{6}\right) \leq C\left\|\widehat{\varphi}_{n}-\widehat{\varphi}\right\|_{H^{1}(\mathbb{R})} \rightarrow 0, \quad \text { as } n \rightarrow \infty,
$$

the convergence to 0 following from case 2 above. This completes the proof of (3.23) when $0<\varepsilon<\frac{1}{2}$. When $\frac{1}{2} \leq \varepsilon<1$, we have, by the same kind of argument,

$$
\omega_{\varepsilon}\left(B_{6}\right) \leq C\left\|\widehat{\varphi}_{n}-\hat{\varphi}\right\|_{H^{\frac{1}{2}+\gamma}(\mathbb{R})} \rightarrow 0, \quad \text { as } n \rightarrow \infty,
$$

where the convergence to 0 now follows from the fact that $1<\frac{1}{2}+\gamma<\frac{3}{2}$ and the previous case. This completes the proof of (3.23) and, with it, establishes Theorem 3.8. 
Remark 3.27. The map $M: m \mapsto M(m) \equiv \prod_{1}^{\infty} m\left(2^{-j}.\right)$, is welldefined, and takes values in $H^{\alpha}(\mathbb{R})$, whenever $m \in \mathcal{F}_{\alpha}$ (see, Chapter 4 of [7]), although, as we saw in Example 3.12, it is not continuous in this case. It is not too hard to modify the proof we just presented to show that, when $m_{n} \rightarrow m$ in $\mathcal{F}_{\alpha}$, then $\widehat{\varphi}_{n} \rightarrow \widehat{\varphi}$ in the local Sobolev space $H_{\text {loc }}^{\alpha}(\mathbb{R})$, where $\widehat{\varphi}_{n}, \widehat{\varphi}$ are the images of $m_{n}, m$ under $M$. That is, if $\alpha=k+\varepsilon, k \in \mathbb{Z}^{+} \cup\{0\}, 0 \leq \varepsilon<1$, for every compact set $K \subset \mathbb{R}$, we have

$$
\lim _{n \rightarrow \infty}\left\{\left\|\widehat{\varphi}_{n}-\widehat{\varphi}\right\|_{L^{2}(K)}+\left\|\widehat{\varphi}_{n}^{(k)}-\widehat{\varphi}^{(k)}\right\|_{L^{2}(K)}+\omega_{\varepsilon, K}\left(\widehat{\varphi}_{n}^{(k)}-\widehat{\varphi}^{(k)}\right)\right\}=0,
$$

where

$$
\omega_{\varepsilon, K}(g)= \begin{cases}{\left[\int_{K} \int_{K}|g(x+h)-g(x)|^{2} d x \frac{d h}{|h|^{1+2 \varepsilon}}\right]^{\frac{1}{2}},} & \text { if } 0<\varepsilon<1 \\ 0, & \text { if } \varepsilon=0 .\end{cases}
$$

In particular, when $\alpha>k+\frac{1}{2}$, the Sobolev Embedding Theorem implies that $\widehat{\varphi}_{n}^{(h)} \rightarrow \widehat{\varphi}^{(h)}$, uniformly on compact sets of $\mathbb{R}, 0 \leq h \leq k$, extending a result of Bonami, Durand and Weiss (Proposition 2.4 of [1] ). A careful proof of these facts appears in section 3.3 of $[\mathbf{3}]$.

\section{Connectivity in the space $\mathcal{W}_{\alpha}$}

In subsection 2.3 we have studied two important objects in the theory of $\alpha$-localized MRA's: the low-pass filters, and the scaling functions. We have seen that the sets $\mathcal{E}_{\alpha}$ and $\mathcal{S}_{\alpha}$, consisting, respectively, of all these elements, are topologically equivalent and arcwise connected. In this section, we shall prove similar properties for wavelets arising from $\alpha$-localized MRA's. First, we recall that these are precisely the wavelets belonging to the set $\mathcal{W}_{\alpha}$ (theorems of Hervé and Lemarié) and, later, we analyze the "phases" associated with these wavelets to obtain the connectivity result in Theorem 1.3. We will also see the relation between the "center of mass" of a wavelet and the homotopy degree of an associated "phase".

\subsection{Wavelets in $\mathcal{W}_{\alpha}$ and $\alpha$-localized MRA's.}

It is well-known that, whenever $\left\{V_{j}\right\}_{j \in \mathbb{Z}}$ is an $\alpha$-localized MRA, with scaling function $\varphi$, and associated low-pass filter $m$, then one can produce a wavelet $\psi^{0}$ by letting

$$
\widehat{\psi}^{0}(\xi)=e^{-i \xi / 2} \overline{m(\xi / 2+\pi)} \widehat{\varphi}(\xi / 2), \quad \xi \in \mathbb{R}
$$


(see Chapter 2 of [5]). Since $\widehat{\varphi} \in H^{\alpha}(\mathbb{R})$ and $m \in H^{\alpha}(\mathbb{T})$, it follows from simple properties of Sobolev spaces that the wavelet $\psi^{0}$ is $\alpha$-localized as well.

It is perhaps not so well-known that, in these conditions, $\varphi$ and, hence, also $\psi^{0}$, belong to a Sobolev space $H^{\varepsilon}(\mathbb{R})$, where the value of $\varepsilon>0$ depends on the spectral radius of a transition operator associated with the filter $m$ (for a proof of this result, due to L. Hervé, we refer the reader to Chapter 4 of [7], or [4]). Thus, every wavelet constructed from an $\alpha$ localized MRA as in (4.1) satisfies the requirements in Definition 1.2 and belongs to $\mathcal{W}_{\alpha}$. We already pointed out this in section 1 .

It is possible to choose other wavelets from the MRA $\left\{V_{j}\right\}$, different to the one in (4.1). That is, functions, $\psi$, for which the system $\{\psi(\cdot-k)\}_{k \in \mathbb{Z}}$ is an orthonormal basis of $W_{0} \equiv V_{0}^{\perp} \cap V_{1}$. The Fourier transform of each of these functions must be given by

$$
\widehat{\psi}(\xi)=\nu(\xi) \widehat{\psi}^{0}(\xi), \quad \xi \in \mathbb{R},
$$

where $\nu$ is unimodular and $2 \pi$-periodic (see Chapter 2 of [5]). Since we are interested in $\psi \alpha$-localized, and the Fourier coefficients of $\nu$ are precisely

$$
\int_{\mathbb{R}} \psi(x) \overline{\psi^{0}(x-k)} d x, \quad k \in \mathbb{Z},
$$

then, by Lemma 3.4, we must have that the "phase" $\nu$ belongs to the Sobolev space $H^{\alpha}(\mathbb{T})$. In other words, if we denote by $\mathcal{M}_{\alpha}$ the topological space consisting of all the unimodular functions in $H^{\alpha}(\mathbb{T})$, it follows from our considerations that the map

$$
\begin{aligned}
T: \mathcal{M}_{\alpha} \times \mathcal{E}_{\alpha} & \longrightarrow \mathcal{W}_{\alpha} \\
(\nu, m) & \longmapsto T(\nu, m)=\psi,
\end{aligned}
$$

where $\psi$ is given by

$$
\widehat{\psi}(\xi)=e^{-i \xi / 2} \overline{\nu(\xi)} \overline{m(\xi / 2+\pi)} \prod_{j=2}^{\infty} m\left(2^{-j} \xi\right), \quad \xi \in \mathbb{R},
$$

is well-defined and continuous. Moreover, the following theorem of Lemarié shows that the "converse" is also true; that is, the map $T$ is onto.

Theorem 4.4 (see [8], or Chapter 3 of $[7]$ ). Let $\frac{1}{2}<\alpha \leq \infty$, and $\psi$ be a wavelet in $\mathcal{W}_{\alpha}$. Then the sequence of spaces

$$
V_{J}=\overline{\operatorname{span}}\left\{\psi_{j, k}: j<J, k \in \mathbb{Z}\right\}, \quad J \in \mathbb{Z},
$$

forms an $\alpha$-localized multiresolution analysis in $L^{2}(\mathbb{R})$. In particular, $\widehat{\psi}$ can be written as in (1.5) for some $\alpha$-localized scaling function $\varphi$, with associated filter $m \in \mathcal{E}_{\alpha}$, and a phase $\nu \in \mathcal{M}_{\alpha}$. 
This characterizes the wavelets in $\mathcal{W}_{\alpha}$ as precisely those arising from $\alpha$-localized multiresolution analyses, and reduces their study to the understanding of filters and phases. Related to the results in this paper, two natural questions can then be asked. One is about the injectivity (and, possibly, openness) of the map $T$. The answer is negative, since there is a whole family of filters and phases that correspond to the same wavelet. A good description of this family can only be given when $\alpha=\infty$, and is presented in the appendix. The other question, directly related to Theorem 1.3, is how from the study of the connectivity in the space $\mathcal{M}_{\alpha}$ we can obtain information about the space $\mathcal{W}_{\alpha}$. This question is more subtle than what one might expect, since the topological space $\mathcal{M}_{\alpha}$ is not connected. We treat it in full detail in the next subsection.

\subsection{The space $\mathcal{M}_{\alpha}$ and the homotopy degree of a wavelet.}

It is very easy to see that the space $\mathcal{M}_{\alpha}$ is not connected when $\alpha>\frac{1}{2}$. Indeed, the functions $\nu_{1} \equiv \mathbf{1}$ and $\nu_{2}(\xi)=e^{i \xi}$, which have different homotopy degrees, cannot be joined with a continuous path of unimodular $2 \pi$-periodic functions, even in the (milder) topology of $C(\mathbb{T})$. We actually may write $\mathcal{M}_{\alpha}$ as the disjoint union of the sets

$$
\mathcal{M}_{\alpha}^{(k)}=\left\{\nu \in \mathcal{M}_{\alpha}: \nu \text { is homotopically equivalent to } e^{i k \xi}\right\}, \quad k \in \mathbb{Z},
$$

each of them being simultaneously open and closed. In the following proposition we collect these and other properties of $\mathcal{M}_{\alpha}$.

Proposition 4.5. Let $\frac{1}{2}<\alpha \leq \infty$ and let $\nu(\xi)=\sum_{\ell \in \mathbb{Z}} c_{\ell} e^{i \ell \xi} \in \mathcal{M}_{\alpha}$. Then:

(i) $\sum_{\ell \in \mathbb{Z}} \ell\left|c_{\ell}\right|^{2} \in \mathbb{Z}$

(ii) For $k \in \mathbb{Z}, \quad \nu \in \mathcal{M}_{\alpha}^{(k)} \quad$ if and only if $k=\sum_{\ell \in \mathbb{Z}} \ell\left|c_{\ell}\right|^{2}$.

Moreover, for every $k \in \mathbb{Z}, \mathcal{M}_{\alpha}^{(k)}$ is a connected open and closed subset of $\mathcal{M}_{\alpha}$. That is, $\mathcal{M}_{\alpha}$ can be written in connected components as the disjoint union $\mathcal{M}_{\alpha}=\cup\left\{\mathcal{M}_{\alpha}^{(k)} \mid k \in \mathbb{Z}\right\}$.

Proof: Suppose first that $\nu \in C^{\infty}(\mathbb{T})$. Then, by Parseval's equality

$$
\begin{aligned}
\frac{1}{2 \pi i} \int_{\nu} \frac{d z}{z} & =\frac{1}{2 \pi i} \int_{0}^{2 \pi} \frac{\nu^{\prime}(t)}{\nu(t)} d t=\frac{1}{2 \pi i} \int_{0}^{2 \pi} \nu^{\prime}(t) \overline{\nu(t)} d t \\
& =\frac{1}{i} \sum_{\ell \in \mathbb{Z}} i \ell c_{\ell} \overline{c_{\ell}}=\sum_{\ell \in \mathbb{Z}} \ell\left|c_{\ell}\right|^{2} .
\end{aligned}
$$


Now, the integral on the left represents the winding number of the curve $\nu$, which is always an integer. Moreover, $\nu$ is homotopically equivalent to $e^{i k \xi}$ if and only if the winding number is $k$. This shows (i) and (ii) when $\alpha=\infty$.

Suppose now that $\frac{1}{2}<\alpha<\infty$. We proceed by density of $\mathcal{M}_{\infty}$ in $\mathcal{M}_{\alpha}$. Indeed, given $\nu \in \mathcal{M}_{\alpha}$, the corresponding sequence of symmetric partial sums of the Fourier series, $s_{N}(\xi)=\sum_{|\ell| \leq N} c_{\ell} e^{i \xi \ell}, N \geq 1$, converges uniformly to $\nu$ and, in particular, satisfies $\left|s_{N}(\xi)\right|>\frac{1}{2}, \xi \in \mathbb{T}$, when $N$ is large enough. Moreover, simple properties of the Banach algebra $H^{\alpha}(\mathbb{T})$ imply that

$$
\nu_{N} \equiv \frac{s_{N}}{\left|s_{N}\right|} \rightarrow \nu \quad \text { in } \quad H^{\alpha}(\mathbb{T})
$$

and, therefore,

$$
\lim _{N \rightarrow \infty} \sum_{\ell \in \mathbb{Z}} \ell\left|c_{\ell}\left(\nu_{N}\right)\right|^{2}=\sum_{\ell \in \mathbb{Z}} \ell\left|c_{\ell}(\nu)\right|^{2}<\infty .
$$

From the previous case (since $\nu_{N} \in C^{\infty}(\mathbb{T})$ ), every term on the left hand side of (4.7) is an integer. Hence, there must exist $k \in \mathbb{Z}$ and $N_{0} \geq 1$, such that $\sum_{\ell \in \mathbb{Z}} \ell\left|c_{\ell}\left(\nu_{N}\right)\right|^{2}=k$, for every $N \geq N_{0}$, establishing the claim made in (i). The equivalence in (ii) follows easily from (4.7) and the fact that each $\mathcal{M}_{\alpha}^{(k)}, k \in \mathbb{Z}$, is closed.

To show the connectivity of $\mathcal{M}_{\alpha}^{(k)}$ note that, every unimodular function $\nu \in H^{\alpha}(\mathbb{T})$, with, say, homotopy degree $k=0$, can be written as

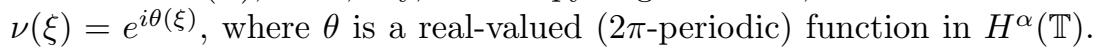
This can be easily seen by using the holomorphic functional calculus in $H^{\alpha}(\mathbb{T})$, and letting $\theta$ be an appropriate choice of the $\log$ of $\nu$. Then, the path $t \mapsto e^{i t \theta}$, connecting $\mathbf{1}$ with $\nu$, takes values in $\mathcal{M}_{\alpha}^{(0)}$, and is continuous in the $H^{\alpha}(\mathbb{T})$ topology, establishing the proposition.

The description of $\mathcal{M}_{\alpha}$ we just gave has immediate consequences on the space of wavelets $\mathcal{W}_{\alpha}$, separating these in different homotopy classes which cannot be connected with a continuous path, even in the topology of $L^{2}(|x| d x)$. Curiously enough, these classes are determined by the "center of mass" of the wavelets, as the following theorem shows:

Theorem 4.8. Let $\frac{1}{2}<\alpha \leq \infty$, and suppose $\psi$ is a wavelet in $\mathcal{W}_{\alpha}$ that can be written as in (1.5). Then,

$$
\int_{\mathbb{R}} x|\psi(x)|^{2} d x=k+\frac{1}{2},
$$

where the integer $k$ corresponds to the homotopy degree of the phase $\nu$. 
Proof: Suppose first that $\alpha=\infty$. Then, using the Plancherel's theorem and the fact that $(x \psi(x))^{\wedge}(\xi)=i \widehat{\psi}^{\prime}(\xi)$, we have

$$
\begin{aligned}
I_{\psi} \equiv \int_{\mathbb{R}} x|\psi(x)|^{2} d x & =\frac{1}{2 \pi} \int_{\mathbb{R}}(x \psi(x))^{\wedge}(\xi) \overline{\widehat{\psi}(\xi)} d \xi \\
& =\frac{i}{2 \pi} \int_{\mathbb{R}} \widehat{\psi}^{\prime}(\xi) \overline{\widehat{\psi}(\xi)} d \xi .
\end{aligned}
$$

Observe that all the integrals above are absolutely convergent under the assumption that $\psi$ has polynomial decay. By taking derivatives in (1.5), we obtain

$$
\begin{aligned}
\widehat{\psi}^{\prime}(\xi) \overline{\widehat{\psi}(\xi)}= & -\frac{i}{2}|\widehat{\psi}(\xi)|^{2}+\bar{\nu}^{\prime}(\xi) \nu(\xi)\left|\widehat{\psi}^{0}(\xi)\right|^{2} \\
& +\frac{1}{2}|\widehat{\varphi}(\xi / 2)|^{2} m(\xi / 2+\pi) \overline{m^{\prime}(\xi / 2+\pi)} \\
& +\frac{1}{2}|m(\xi / 2+\pi)|^{2} \widehat{\varphi}^{\prime}(\xi / 2) \overline{\widehat{\varphi}(\xi / 2)},
\end{aligned}
$$

where the function $\psi^{0}$ in the second summand denotes the wavelet whose Fourier transform is given by the right hand side of (4.1). Substituting this expression in (4.9), and periodizing, or changing variables, in the new integrals, we obtain:

$$
\begin{aligned}
I_{\psi}= & \frac{1}{4 \pi}\|\widehat{\psi}\|_{L^{2}(\mathbb{R})}^{2}+\frac{i}{2 \pi} \int_{\mathbb{T}} \bar{\nu}^{\prime}(\xi) \nu(\xi) \sum_{\ell \in \mathbb{Z}}\left|\widehat{\psi}^{0}(\xi+2 \ell \pi)\right|^{2} d \xi \\
& +\frac{i}{2 \pi}\left\{\int_{\mathbb{R}}|\widehat{\varphi}(\xi)|^{2} m(\xi+\pi) \overline{m^{\prime}(\xi+\pi)} d \xi+\int_{\mathbb{R}}|m(\xi+\pi)|^{2} \widehat{\varphi}^{\prime}(\xi) \overline{\hat{\varphi}(\xi)} d \xi\right\} \\
= & \frac{1}{2}+\operatorname{deg}(\nu)+\frac{i}{2 \pi}\{A+B\},
\end{aligned}
$$

where in the second equality we have used that $\sum_{\ell \in \mathbb{Z}}\left|\widehat{\psi}^{0}(\xi+2 \ell \pi)\right|^{2}=1$ (because $\psi^{0}$ is a wavelet), and the formula in (4.6) for the winding number of $\nu$. We will show that the integrals corresponding to $A$ and $B$ in the brackets above are conjugate of each other, and this will establish our result (since $I_{\psi}$ is a real number). Indeed, by a periodization argument, we can write:

$$
\begin{aligned}
A & =\sum_{\ell \in \mathbb{Z}} \int_{\mathbb{T}}|\widehat{\varphi}(\xi+2 \ell \pi)|^{2} m(\xi+\pi) \overline{m^{\prime}(\xi+\pi)} d \xi \\
& =\int_{\mathbb{T}} m(\xi+\pi) \overline{m^{\prime}(\xi+\pi)} d \xi=\int_{\mathbb{T}} m(\xi) \overline{m^{\prime}(\xi)} d \xi
\end{aligned}
$$


where in the second equality we have used $\sum_{\ell \in \mathbb{Z}}|\widehat{\varphi}(\xi+2 \ell \pi)|^{2}=1$. On the other hand, from $|m(\xi)|^{2}+|m(\xi+\pi)|^{2}=1$, it follows that:

$$
\begin{aligned}
B & =\int_{\mathbb{R}}|m(\xi+\pi)|^{2} \widehat{\varphi}^{\prime}(\xi) \overline{\hat{\varphi}(\xi)} d \xi \\
& =\int_{\mathbb{R}} \widehat{\varphi}^{\prime}(\xi) \overline{\widehat{\varphi}(\xi)} d \xi-\int_{\mathbb{R}}|m(\xi)|^{2} \widehat{\varphi}^{\prime}(\xi) \overline{\hat{\varphi}(\xi)} d \xi .
\end{aligned}
$$

Now, differentiating in the scaling equation (3.2), and changing variables, we obtain

$$
\begin{aligned}
\int_{\mathbb{R}} \widehat{\varphi}^{\prime}(\xi) \overline{\hat{\varphi}(\xi)} d \xi & =\int_{\mathbb{R}} m^{\prime}(\xi) \overline{m(\xi)}|\widehat{\varphi}(\xi)|^{2} d \xi+\int_{\mathbb{R}}|m(\xi)|^{2} \widehat{\varphi}^{\prime}(\xi) \overline{\hat{\varphi}(\xi)} d \xi \\
& =\int_{\mathbb{T}} m^{\prime}(\xi) \overline{m(\xi)} d \xi+\int_{\mathbb{R}}|m(\xi)|^{2} \widehat{\varphi}^{\prime}(\xi) \overline{\widehat{\varphi}(\xi)} d \xi .
\end{aligned}
$$

Thus, we can write $B$ as:

$$
B=\int_{\mathbb{T}} m^{\prime}(\xi) \overline{m(\xi)} d \xi=\bar{A} .
$$

This shows that $I_{\psi}=\frac{1}{2}+\operatorname{deg}(\nu)$, and establishes the case $\alpha=\infty$.

For the general case, $\alpha>\frac{1}{2}$, we proceed by density of $\mathcal{W}_{\infty}$ in $\mathcal{W}_{\alpha}$. Let us write $\psi \in \mathcal{W}_{\alpha}$ as:

$$
\widehat{\psi}(\xi)=e^{-i \frac{\xi}{2}} \overline{\nu(\xi)} \overline{m(\xi / 2+\pi)} \widehat{\varphi}(\xi / 2),
$$

where $\nu \in \mathcal{M}_{\alpha}, m \in \mathcal{E}_{\alpha}$ and $\widehat{\varphi}(\xi)=\prod_{j=1}^{\infty} m\left(2^{-j} \xi\right) \in \mathcal{S}_{\alpha}$. Suppose that $\nu$ is homotopically equivalent to $e^{i k \xi}$. Then, in the same way as in the proof of Proposition 4.5, we can find a sequence $\left\{\nu_{N}\right\}_{N=1}^{\infty} \subset \mathcal{M}_{\infty}^{(k)}$ such that $\nu_{N} \rightarrow \nu$ in $H^{\alpha}(\mathbb{T})$. By Remark 2.15, we can find another sequence $\left\{m_{N}\right\}_{N=1}^{\infty} \subset \mathcal{E}_{\infty}$ such that $m_{N} \rightarrow m$ in $H^{\alpha}(\mathbb{T})$. Then, by the considerations in subsection 4.1 and Theorem 3.8, the functions $\psi_{N}$ defined by

$$
\widehat{\psi}_{N}(\xi)=e^{-i \frac{\xi}{2}} \overline{\nu_{N}(\xi)} \overline{m_{N}(\xi / 2+\pi)} \prod_{j=2}^{\infty} m_{N}\left(2^{-j} \xi\right), \quad \xi \in \mathbb{R}, N \geq 1,
$$

are wavelets in $\mathcal{W}_{\infty}^{(k)}$ and $\widehat{\psi}_{N} \rightarrow \widehat{\psi}$ in $H^{\alpha}(\mathbb{R})$. This, together with the previous case, implies that

$$
\int_{\mathbb{R}} x|\psi(x)|^{2} d x=\lim _{N \rightarrow \infty} \int_{\mathbb{R}} x\left|\psi_{N}(x)\right|^{2} d x=k+\frac{1}{2},
$$

completing the proof of the theorem. 
The proof of Theorem 1.3: Theorem 1.3 follows almost immediately from our previous results. Indeed, we have shown formula (1.4) in Theorem 4.8 , which clearly implies that the spaces $\mathcal{W}_{\alpha}^{(k)}, k \in \mathbb{Z}$, are open and closed. Moreover, from the ontoness of the map $T$ in (4.2), it follows that $T\left(\mathcal{M}_{\alpha}^{(k)} \times \mathcal{E}_{\alpha}\right)=\mathcal{W}_{\alpha}^{(k)}, k \in \mathbb{Z}$, and since $\mathcal{M}_{\alpha}^{(k)}$ and $\mathcal{E}_{\alpha}$ are connected, $\mathcal{W}_{\alpha}^{(k)}$ must be also, completing the proof of Theorem 1.3.

\section{Appendix: More about the phase}

In the previous section we saw that, as a consequence of Lemarié's Theorem, every wavelet $\psi \in \mathcal{W}_{\alpha}$ can be written as in (1.5) for a suitable triad of functions $\nu, m$ and $\varphi$. We have seen, further, that if $\psi \in \mathcal{W}_{\alpha}^{(k)}$, then $\nu$ must have homotopy degree $k$. One can ask whether an apropriate choice of the filter $m$, will give a "simple" expression for the phase, like that in (4.1) (or with $\nu(\xi)=e^{i k \xi}$, when $k \neq 0$ ). Rephrasing the question with the notation in section 4 , given $\psi \in \mathcal{W}_{\alpha}^{(k)}$, is there a representative of the form $\left(e^{i k \xi}, m\right)$ in the fiber $T^{-1}(\psi)$ ? In the case $\alpha=\infty$ we have the following affirmative result, due to Lemarié:

Proposition 5.1 (see Théorème 4 in [8]). Let $\psi \in \mathcal{W}_{\infty}^{(k)}$. Then, there exists a filter $m \in \mathcal{E}_{\infty}$, and a unimodular constant $c \in \mathbb{C}$ such that

$$
\widehat{\psi}(\xi)=c e^{-i \frac{\xi}{2}} e^{-i k \xi} \overline{m(\xi / 2+\pi)} \prod_{j=2}^{\infty} m\left(2^{-j} \xi\right), \quad \xi \in \mathbb{R} .
$$

If $\left(c^{\sharp}, m^{\sharp}\right) \in S^{1} \times \mathcal{E}_{\infty}$ is another solution to (5.2), then there exists an integer $M \in \mathbb{Z}$ such that $c^{\sharp}=(-1)^{M} c$ and $m^{\sharp}(\xi)=e^{i M \xi} m(\xi), \xi \in \mathbb{T}$.

In particular, if we let the equivalence relation " $~ "$ in $S^{1} \times \mathcal{E}_{\infty}$ :

$$
(c, m) \sim\left(c^{\sharp}, m^{\sharp}\right) \quad \Longleftrightarrow \quad \exists M \in \mathbb{Z}: c=(-1)^{M} c^{\sharp}, m(\xi)=e^{i M \xi} m^{\sharp}(\xi)
$$

and we introduce the quotient topology in $\left(S^{1} \times \mathcal{E}_{\infty}\right) / \sim$, then the map:

$$
\begin{aligned}
\left.\bar{T}_{k}: \quad S^{1} \times \mathcal{E}_{\infty}\right) / \sim & \longrightarrow \mathcal{W}_{\infty}^{(k)} \\
{[(c, m)] } & \longmapsto T_{k}(c, m)=\psi,
\end{aligned}
$$

where $\psi$ is given by (5.2), is a continuous bijection of topological spaces. It would be of interest to know whether $\bar{T}_{k}$ is open, since this would provide a characterization for the topology of $\mathcal{W}_{\infty}$ in terms of $\mathcal{E}_{\infty}$. At this point, we do not have a complete answer to this question. 
When we move to the case $\frac{1}{2}<\alpha<\infty$, the situation changes dramatically. To be more precise, suppose we have a function $\nu \in H^{\alpha}(\mathbb{T})$, unimodular, and with, say, homotopy degree 0 . Consider, then, the following functional equation:

$$
\nu(2 \xi)=\mu(2 \xi) \overline{\mu(\xi) \mu(\xi+\pi)}, \quad \xi \in \mathbb{T},
$$

where $\mu$ is the unknown, to be in the space $\mathcal{M}_{\beta}^{(0)}$, for the largest possible $\beta$ (we would like $\beta=\alpha$ ). If we could solve this equation, then, by letting

$$
\tilde{m}(\xi)=\mu(2 \xi) \overline{\mu(\xi)} m(\xi), \quad \xi \in \mathbb{R},
$$

we obtain a "nice" representation for the wavelet $\psi$ in (4.3), since:

$$
\begin{aligned}
\widehat{\psi}(\xi) & =c e^{-i \frac{\xi}{2}} \overline{\mu(\xi)} \mu(\xi / 2+\pi) \mu(\xi / 2) \overline{m(\xi / 2+\pi)} \prod_{j=2}^{\infty} m\left(2^{-j} \xi\right) \\
& =c e^{-i \frac{\xi}{2}} \overline{\tilde{m}(\xi / 2+\pi)} \prod_{j=2}^{\infty} \tilde{m}\left(2^{-j} \xi\right) .
\end{aligned}
$$

If the solution $\mu$ belongs to the Sobolev space $H^{\beta}(\mathbb{T})$, then the new filter $\tilde{m}$ is in $\mathcal{E}_{\beta}$, and we have a partial answer to our question. Moreover, if we could take $\beta=\alpha$, then we would have a natural extension of Proposition 5.1 to the $\alpha$-localized situation. Unfortunately, this case is not attainable when $\alpha<\infty$, the most we can say being contained in the following result:

Theorem 5.4. Let $\frac{1}{2}<\alpha \leq \infty$ and $\nu \in \mathcal{M}_{\alpha}^{(0)}$ and write $\nu(\xi)=$ $e^{i \theta(\xi)}$, for some real-valued $\theta(\xi)=\sum_{\ell \in \mathbb{Z}} \theta_{\ell} e^{i \ell \xi} \in H^{\alpha}(\mathbb{T})$. Consider the functional equation in (5.3). Then,

(i) If $1<\alpha \leq \infty$, (5.3) has a unique solution $\mu(\xi)=e^{i \gamma(\xi)} \in$ $\mathcal{M}_{1}^{(0)}$ given by $\gamma(\xi)=\sum_{\ell \in \mathbb{Z}} \gamma_{\ell} e^{i \ell \xi}$, where $\gamma_{0}=-\theta_{0}$ and $\gamma_{\ell}=$ $\sum_{j=0}^{\infty} 2^{j} \theta_{2^{j} \ell}, \ell \neq 0$. Moreover, in this case $\mu \in \cap_{\beta<\alpha} H^{\beta}(\mathbb{T})$. If $\alpha \neq \infty$, there are examples for which the solution $\mu \notin H^{\alpha}(\mathbb{T})$.

(ii) If $\frac{1}{2}<\alpha \leq 1$, (5.3) has infinitely many solutions $\mu \in \cap_{\beta<\alpha} \mathcal{M}_{\beta}^{(0)}$. There are examples for which none of the solutions belong to $H^{\alpha}(\mathbb{T})$.

As a corollary we obtain the following extension of Proposition 5.1: 
Corollary 5.5. Let $\frac{1}{2}<\alpha \leq \infty, k \in \mathbb{Z}$ and $\psi \in \mathcal{W}_{\alpha}^{(k)}$. Then, there exists a low-pass filter $m \in \cap_{\beta<\alpha} \mathcal{E}_{\beta}$ such that (5.2) holds for some unimodular constant $c \in \mathbb{C}$. Furthermore, if $(c, m)$ and $\left(c^{\sharp}, m^{\sharp}\right)$ are two solutions to (5.2) such that $m, m^{\sharp} \in H^{1}(\mathbb{T})$, then, there exists an integer $M \in \mathbb{Z}$ such that $c^{\sharp}=(-1)^{M} c$ and $m^{\sharp}(\xi)=e^{i M \xi} m(\xi)$.

To prove the theorem we rewrite the functional equation (5.3) in terms of the real-valued functions $\theta$ and $\gamma$, and their Fourier coefficients. In this setting, it is easy to check that (5.3) is equivalent to solve the difference equation:

$$
\left\{\begin{array}{l}
\theta_{\ell}=\gamma_{\ell}-2 \gamma_{2 \ell}, \quad \ell \neq 0 \\
\theta_{0}=-\gamma_{0}+2 K \pi
\end{array}\right.
$$

for some fixed constant $K \in \mathbb{Z}$ (that we will assume equal to 0 ), and with the additional assumption $\overline{\gamma_{\ell}}=\gamma_{-\ell}, \ell \in \mathbb{Z}$ (so that $\gamma$ is real-valued).

By iteration in (5.6), we obtain the following sequence as candidate to solve the equation:

$$
\gamma_{\ell}= \begin{cases}\sum_{j=0}^{\infty} 2^{j} \theta_{2^{j} \ell}, & \text { if } \ell \neq 0 \\ -\theta_{0}, & \text { if } \ell=0\end{cases}
$$

Assume now that $\alpha>1$. Then since $\theta \in H^{\alpha}(\mathbb{T})$ (and, hence, $\theta_{\ell} \in o\left(|\ell|^{\alpha}\right)$, as $|\ell| \rightarrow \infty)$, it follows that the series $\sum_{j=0}^{\infty} 2^{j} \theta_{2^{j} \ell}$ converges absolutely for every $\ell \neq 0$, providing $\left\{\gamma_{\ell}\right\}$ a solution to the difference equation (5.6). We claim, in addition, that $\gamma$ belongs to $H^{\beta}(\mathbb{T})$, for every $\beta<\alpha$. Indeed,

$$
\begin{aligned}
\sum_{\ell \neq 0}\left|\gamma_{\ell}\right|^{2}|\ell|^{2 \beta} & \leq \sum_{\ell \neq 0}\left(\sum_{j=0}^{\infty} 2^{j}\left|\theta_{2^{j} \ell}\right|\right)^{2}|\ell|^{2 \beta} \\
& \leq \sum_{\ell \neq 0}\left(\sum_{j=0}^{\infty} \frac{1}{2^{2 j(\alpha-1)}}\right)\left(\sum_{j=0}^{\infty} 2^{2 j \alpha}\left|\theta_{2^{j} \ell}\right|^{2}\right)|\ell|^{2 \beta} \\
& =\frac{2^{2(\alpha-1)}}{2^{2(\alpha-1)}-1} \sum_{p=0}^{\infty} \sum_{m \in 2 \mathbb{Z}+1} \sum_{j=0}^{\infty}\left(2^{j+p}|m|\right)^{2 \alpha}\left|\theta_{2^{j+p}}\right|^{2} \frac{1}{\left|2^{p} m\right|^{2(\alpha-\beta)}} \\
& \leq \frac{4^{\alpha-1}}{4^{\alpha-1}-1} \sum_{p=0}^{\infty}\left(\sum_{s \in \mathbb{Z}}|s|^{2 \alpha}\left|\theta_{s}\right|^{2}\right) \frac{1}{2^{2(\alpha-\beta) p}} \\
& \leq C(\alpha, \beta) \sum_{s \in \mathbb{Z}}|s|^{2 \alpha}\left|\theta_{s}\right|^{2} .
\end{aligned}
$$


This shows the existence part of the theorem (when $\alpha>1$ ). For the uniqueness note that, if we assume $\left\{\tilde{\gamma}_{\ell}\right\} \in H^{1}(\mathbb{T})$, then $\gamma_{\ell}=o(|\ell|)$, as $|\ell| \rightarrow \infty$, and iterating the difference equation (5.6) we obtain, for $\ell \neq 0$,

$$
\tilde{\gamma}_{\ell}=\sum_{j=0}^{n} 2^{j} \theta_{2^{j} \ell}+2^{n+1} \tilde{\gamma}_{2^{n+1} \ell} \rightarrow \sum_{j=0}^{\infty} 2^{j} \theta_{2^{j} \ell}, \quad \text { as } n \rightarrow \infty,
$$

showing that $\tilde{\gamma}_{\ell}=\gamma_{\ell}$.

With respect to the cases $\frac{1}{2}<\alpha \leq 1$, note that when $\theta \in H^{1}(\mathbb{T})$ the series $\sum_{j=0}^{\infty} 2^{j} \theta_{2^{j}}$ might be divergent (see Example 5.9 below). To construct a solution to the difference equation (5.6) we iterate "backwards", assuming a priori that $\gamma_{2 \ell+1}=0, \ell \in \mathbb{Z}$. Then, we define the rest of the coefficients $\gamma_{\ell}$ by:

$$
\gamma_{\ell}= \begin{cases}-\theta_{0}, & \text { if } \ell=0 \\ 0, & \text { if } \ell \in 2 \mathbb{Z}+1 \\ -\sum_{j=1}^{n} 2^{-j} \theta_{2^{n-j} p}, & \text { if } \ell=2^{n} p, n \geq 1, p \in 2 \mathbb{Z}+1 .\end{cases}
$$

An easy computation shows that $\gamma_{\ell}=\theta_{\ell}+2 \gamma_{2 \ell}, \ell \in \mathbb{Z}$. We claim, further, that $\gamma \in H^{\beta}(\mathbb{T})$, for every $\beta<\alpha$. Indeed,

$$
\begin{aligned}
\sum_{\ell \neq 0}|\ell|^{2 \beta}\left|\gamma_{\ell}\right|^{2} & =\sum_{n=1}^{\infty} \sum_{p \in 2 \mathbb{Z}+1}\left|2^{n} p\right|^{2 \beta}\left|\sum_{j=1}^{n} 2^{-j} \theta_{2^{n-j} p}\right|^{2} \\
& \leq \sum_{n=1}^{\infty} \sum_{p \in 2 \mathbb{Z}+1}\left|2^{n} p\right|^{2 \beta}\left(\sum_{j=1}^{n} 2^{-2 j(1-\alpha)}\right)\left(\sum_{j=1}^{n} 2^{-2 j \alpha}\left|\theta_{2^{n-j} p}\right|^{2}\right) \\
& \leq \sum_{n=1}^{\infty} \sum_{p \in 2 \mathbb{Z}+1} \sum_{j=1}^{n} 2^{2 n \beta}|p|^{2 \beta} n 2^{-2 j \alpha}\left|\theta_{2^{n-j} p}\right|^{2} \\
& =\sum_{n=1}^{\infty} \sum_{p \in 2 \mathbb{Z}+1} \sum_{j=1}^{n}\left|2^{n-j} p\right|^{2 \alpha}\left|\theta_{2^{n-j} p}\right|^{2} \frac{1}{|p|^{2(\alpha-\beta)}} \frac{n}{2^{2 n(\alpha-\beta)}} \\
& \leq \sum_{n=1}^{\infty} \sum_{p \in 2 \mathbb{Z}+1} \sum_{s=0}^{n-1}\left|2^{s} p\right|^{2 \alpha}\left|\theta_{2^{s} p}\right|^{2} \frac{n}{2^{2 n(\alpha-\beta)}} \\
& =\sum_{s=0}^{\infty} \sum_{p \in 2 \mathbb{Z}+1}\left|2^{s} p\right|^{2 \alpha}\left|\theta_{2^{s} p}\right|^{2} \sum_{n=s+1}^{\infty} \frac{n}{2^{2 n(\alpha-\beta)}} \\
& \leq C_{\alpha, \beta} \sum_{r \in \mathbb{Z}}|r|^{2 \alpha}\left|\theta_{r}\right|^{2}<\infty .
\end{aligned}
$$


The non-uniqueness of the solutions to (5.6), in this case, can be guessed from the freedom to choose the odd coefficients $\gamma_{2 \ell+1}$. A more constructive proof is the following. Consider the lacunary sequence

$$
\gamma_{\ell}= \begin{cases}2^{-j}, & \text { if }|\ell|=2^{j}, j \geq 0 \\ 0, & \text { otherwise. }\end{cases}
$$

Note that $\sum_{\ell \in \mathbb{Z}}\left|\gamma_{\ell}\right|^{2}\left(1+|\ell|^{2}\right)^{\beta}<\infty$, for all $\beta<1$, while $\gamma_{\ell}=2 \gamma_{2 \ell}, \ell \neq 0$, and $\gamma_{0}=0$. We have then obtained a non-trivial solution in $\cap_{\beta<1} H^{\beta}(\mathbb{T})$ to the difference equation (5.6) when $\theta \equiv 0$. In fact, multiplying $\left\{\gamma_{\ell}\right\}$ by any real constant we obtain infinitely many others. From here it follows immediately that (5.3) has infinitely many solutions $\mu \in \cap_{\beta<\alpha} \mathcal{M}_{\beta}^{(0)}$, when $\alpha \leq 1$.

We now turn to the "sharpness" of our results. In the example below we give a choice of $\theta \in H^{\alpha}(\mathbb{T})$ for which the solutions to (5.6) do not belong to $H^{\alpha}(\mathbb{T})$.

Example 5.9. We define the following lacunary sequence $\left\{\theta_{\ell}\right\}_{\ell \in \mathbb{Z}}$ :

$$
\theta_{\ell}= \begin{cases}\frac{1}{2^{\alpha 2^{j} j}}, & \text { if }|\ell|=2^{2^{j}}, j \geq 1 \\ 0, & \text { otherwise. }\end{cases}
$$

Note that $\sum_{\ell \in \mathbb{Z}}\left|\theta_{\ell}\right|^{2}|\ell|^{2 \alpha}=\sum_{j \geq 1} \frac{1}{j^{2}}<\infty$. Suppose $\alpha \geq 1$. Then, if we expect the solutions $\gamma$ to $(5.6)$ to belong to the space $H^{\alpha}(\mathbb{T})$, we must have that $\gamma_{\ell}=o(|\ell|)$, as $|\ell| \rightarrow \infty$, and the same type of argument as in (5.7) would imply that $\gamma_{\ell}=\sum_{j=0}^{\infty} 2^{j} \theta_{2^{j} \ell}$. In the case $\alpha=1$, this is already a contradiction since $\gamma_{1}=\sum_{j=0}^{\infty} 2^{j} \theta_{2^{j}}=\sum_{r=1}^{\infty} \frac{1}{r}=\infty$. For the case $\alpha>1$, we have

$$
\begin{aligned}
\sum_{\ell=1}^{\infty}\left|\gamma_{\ell}\right|^{2}|\ell|^{2 \alpha} & =\sum_{m=1}^{\infty} 2^{2 m \alpha}\left|\gamma_{2^{m}}\right|^{2}=\sum_{m=1}^{\infty} 2^{2 m \alpha}\left|\sum_{j=0}^{\infty} 2^{j} \theta_{2^{j+m}}\right|^{2} \\
& =\sum_{m=1}^{\infty} 2^{2(\alpha-1) m}\left|\sum_{j=m}^{\infty} 2^{j} \theta_{2^{j}}\right|^{2} \\
& \geq \sum_{m=2}^{\infty} 2^{2(\alpha-1) m}\left|\sum_{r=\left[\log _{2} m\right]+1}^{\infty} 2^{2^{r}} \frac{1}{2^{\alpha 2^{r} r}}\right|^{2} \\
& \geq C \sum_{m=2}^{\infty} 2^{2(\alpha-1) m}\left|\frac{2^{m}}{2^{\alpha m} \log _{2} m}\right|^{2}=C \sum_{m=2}^{\infty} \frac{1}{\left(\log _{2} m\right)^{2}}=\infty
\end{aligned}
$$

which is also a contradiction. This completes the proof of part (i) in Theorem 5.4, and the case $\alpha=1$ in part (ii). 
Suppose now that, with the same sequence $\left\{\theta_{\ell}\right\}$, we let instead $\frac{1}{2}<$ $\alpha<1$, and assume there is a solution $\gamma \in H^{\alpha}(\mathbb{T})$ to the difference equation (5.6). Then, by iteration, we must have that $\gamma_{2^{n}}=\frac{1}{2^{n}} \gamma_{1}-$ $\sum_{j=1}^{n} \frac{1}{2^{j}} \theta_{2^{n-j}}, n \geq 1$. Now, if $\gamma_{1}=0$, then

$$
\begin{aligned}
\sum_{\ell=1}^{\infty}|\ell|^{2 \alpha}\left|\gamma_{\ell}\right|^{2} & \geq \sum_{n=3}^{\infty} 2^{2 n \alpha}\left|\sum_{j=1}^{n} 2^{-j} \theta_{2^{n-j}}\right|^{2} \\
& =\sum_{n=3}^{\infty} \frac{1}{2^{2 n(1-\alpha)}}\left|\sum_{r=1}^{\left[\log _{2}(n-1)\right]} 2^{2^{r}} \frac{1}{2^{\alpha 2^{r} r}}\right|^{2} \\
& \geq C \sum_{n=3}^{\infty} \frac{1}{2^{2 n(1-\alpha)}}\left|\frac{2^{(1-\alpha) n}}{\log _{2} n}\right|^{2}=C \sum_{n=3}^{\infty} \frac{1}{\left(\log _{2} n\right)^{2}}=\infty
\end{aligned}
$$

which is a contradiction. On the other hand, if $\gamma_{1} \neq 0$, then

$$
\begin{aligned}
& \sum_{n=3}^{\infty} 2^{2 n \alpha}\left|\gamma_{2^{n}}\right|^{2}= \sum_{n=3}^{\infty} \frac{1}{2^{2 n(1-\alpha)}}\left|\gamma_{1}-\sum_{j=1}^{n} 2^{n-j} \theta_{2^{n-j}}\right|^{2} \\
& \geq \sum_{n=3}^{\infty} \frac{1}{2^{2 n(1-\alpha)}}\left\{\left|\gamma_{1}\right|^{2}+\left|\sum_{j=1}^{n} 2^{n-j} \theta_{2^{n-j}}\right|^{2}-2\left|\gamma_{1}\right|\left|\sum_{j=1}^{n} 2^{n-j} \theta_{2^{n-j}}\right|\right\} \\
& \geq\left|\gamma_{1}\right|^{2} \sum_{n=3}^{\infty} \frac{1}{2^{2 n(1-\alpha)}} \\
&+C \sum_{n=3}^{\infty} \frac{1}{\left(\log _{2} n\right)^{2}}-2\left|\gamma_{1}\right| \widetilde{C} \sum_{n=3}^{\infty} \frac{1}{2^{n(1-\alpha)} \log _{2} n}=+\infty
\end{aligned}
$$

where in the last inequality we have used that $\sum_{j=1}^{n} 2^{n-j} \theta_{2^{n-j}} \simeq \frac{2^{n(1-\alpha)}}{\log _{2} n}$. But this is also a contradiction. Thus, we have completed the second part of (ii), and established Theorem 5.4.

Final comments. Before ending, we would like to mention the work done by other authors related to the connectivity of wavelets since the article [1] appeared in the literature. X. Dai, D. Larson and G. Weiss, together with their collaborators, have studied this question from a slightly different point of view. They considered MRA wavelets, but with no localization or smoothness assumption on them, and obtained connecting 
paths continuous only in the topology of $L^{2}(\mathbb{R})$. The treatment of filters and phases differs from ours, and no homotopy impediments appear in this case. A complete exposition of their results can be found in [12]. The paper [10] contains the first general connectivity result involving non-MRA wavelets. However, the question of whether the set of all wavelets is connected still remains open.

\section{References}

1. A. Bonami, S. Durand And G. Weiss, Wavelets obtained by continuous deformations of the Haar wavelet, Rev. Mat. Iberoamericana 12(1) (1996), 1-18.

2. A. Cohen, "Ondelettes et traitement numérique du signal," Rech. Math. Appl. 25, 1992.

3. G. GARRIGós, The characterization of wavelets and related functions and the connectivity of $\alpha$-localized wavelets on $\mathbb{R}$, Ph. D. Thesis, Washington University (1998). (Available at http://www.math.wustl.edu/ gustavo).

4. L. Hervé, Méthodes d'opérateurs quasi-compacts en analyse multirésolution, applications à la construction de bases d'ondelettes et à l'interpolation, Thèse, Laboratoire de Probabilités, Université de Rennes-I (1992).

5. E. Hernández and G. L. Weiss, "A First Course on Wavelets," CRC Press, 1996.

6. E. Hernández, X. Wang and G. Weiss, Smoothing Minimally Supported Frequency Wavelets. Part I, J. Fourier Anal. Appl. 2(4) (1996), 329-340.

7. J. P. Kahane and P. G. Lemarié-Rieusset, "Fourier Series and Wavelets," Gordon \& Breach Publishers, 1995.

8. P. G. Lemarié-Rieusset, Projecteurs invariants, matrices de dilatation, ondelettes et analyses multi-résolution, Rev. Mat. Iberoamericana 10(2) (1994), 283-347.

9. P. G. Lemarié-Rieusset, Fonctions d'échelle interpolantes, polynômes de Bernstein et ondelettes non stationnaires, Rev. Mat. Iberoamericana 13(1) (1997), 91-188.

10. D. M. SpeEgle, The $S$-elementary wavelets are path-connected, Proc. Amer. Math. Soc. 127(1) (1999), 223-233.

11. L. F. Villemoes, Energy moments in time and frequency for twoscale difference equation solutions and wavelets, SIAM J. Math. Anal. 23(6) (1992), 1519-1543. 
12. The Wutam Consortium, Basic Properties of Wavelets, $J$. Fourier Anal. Appl. 4(4) (1998), 575-594.

\author{
Dipartimento di Matematica \\ Politecnico di Torino \\ Corso Duca degli Abruzzi, 24 \\ 10129 Torino \\ ITALY \\ e-mail: gustavo@calvino.polito.it
}

Primera versió rebuda el 27 d'octubre de 1998 , darrera versió rebuda el 14 de gener de 1999 